\title{
Discovering the lichen diversity of a remote tropical island: working list of species collected on Reunion (Mascarene archipelago, Indian Ocean)
}

\author{
Pieter P. G. van Den Boom, Maarten Brand, Damien Ertz, Klaus Kalb, Nicolas \\ Magain, Didier Masson, Ulf Schiefelbein, Harrie J. M. Sipman \& \\ Emmanuël SÉRUSIAUX ${ }^{1}$
}

\begin{abstract}
Boom, P. P. G., Brand, M., Ertz, D., Kalb, K., Magain, N., Masson, D., Schiefelbein, U., Sipman, H. J. M. \& SÉrusiaux, E. 2011. Discovering the lichen diversity of a remote tropical island: working list of species collected on Reunion (Mascarene archipelago, Indian Ocean). - Herzogia 24: 325-349.

463 lichen taxa are reported from the island of Reunion (Mascarene archipelago) in the Indian Ocean. This report is based on extensive collections recently made by the authors or hosted in B and LG. A total of c. 600 species is estimated for the number of lichenized fungi present on the island; although this is a low figure for a varied tropi$\mathrm{cal}$ area, this is plausible due to its small size and remoteness. The significance of the lichen diversity of the island lays in its endemics, which need further study for a reliable assessment, and in the occurrence of a poorly known southern hemisphere element ("austral" species). The discovery of the following in Reunion is of special interest as it considerably enlarges their distribution range: Anzia corallophora, Caloplaca caesiosorediata, Coccocarpia delicatula, Fuscopannaria praetermissa, Gomphillus morchelloides, G. pedersenii, Gyalideopsis bispora, Heterodermia koyana, Hypotrachyna evansii, Lithogyalideopsis zeylandica, Lobaria asperula, Megaloblastenia marginiflexa var. dimota, Menegazzia capitata, Myeloconis erumpens and Pannaria squamulosa.
\end{abstract}

Zusammenfassung: van den Boom, P. P. G., Brand, M., Ertz, D., Kalb, K., Magain, N., Masson, D., Schiefelbein, U., Sipman, H. J. M. \& SÉrusiaux, E. 2011. Die Entdeckung der Flechtenvielfalt einer entlegenen Insel: eine Arbeitsliste von auf Reunion (Maskarenen, Indischer Ozean) gesammelten Arten. - Herzogia 24: 325-349.

Von der Insel Reunion (Maskarenen) im Indischen Ozean werden 463 Flechtentaxa gemeldet. Dieses Ergebnis basiert auf umfangreichen Aufsammlungen, die von den Autoren in den letzten Jahren gemacht wurden oder in B und LG vorhanden sind. Mit ca. 600 Arten als angemessener Schätzwert für die Gesamtzahl lichenisierter Pilze auf der Insel wird gerechnet. Dies mag für ein sehr vielgestaltiges, tropisches Gebiet wenig erscheinen, ist aber plausibel, wenn man die geringe Größe und Abgeschiedenheit in Betracht zieht. Die Bedeutung der Artenvielfalt der Insel liegt jedoch einerseits in ihrem Endemismus, wofür noch mehr Untersuchungen nötig sind, um zu einer verlässlichen Bewertung zu gelangen, andererseits im Vorkommen eines noch nur wenig bekannten, südhemisphärischen Elements (sogenannte „australe“ Arten). Die Entdeckung folgender Spezies in Reunion ist von besonderem Interesse, da deren Verbreitungsgebiet hiermit beträchtlich erweitert wird: Anzia corallophora, Caloplaca caesiosorediata, Coccocarpia delicatula, Fuscopannaria praetermissa, Gomphillus morchelloides, G. pedersenii, Gyalideopsis bispora, Heterodermia koyana, Heterodermia koyana, Hypotrachyna evansii, Lithogyalideopsis zeylandica, Lobaria asperula, Megaloblastenia marginiflexa var. dimota, Menegazzia capitata, Myeloconis erumpens und Pannaria squamulosa.

Key words: Lichenized Ascomycetes, austral element, Bory de St-Vincent.

$\overline{{ }^{1} \text { corresponding author }}$ 


\section{Introduction}

In the most documented estimation of the number of lichenized fungi present worldwide, LÜCKING et al. (2009) argued for a total figure of 28,000 species, only half of which had been formally described. Indeed, much remains to be done to assess the overall $\alpha$-diversity of lichenized fungi, as masterly demonstrated by the recent initiative by a previously unrivalled team of scientists in describing a set of 100 new species from all biomes in the world (LuMBSCH et al. 2011).

Further, the breakthrough of easy access to DNA sequences, and use of powerful statistical methods to make the most out of those large datasets within a phylogenetical context, has sharpened the generic assignments of many species (SÉRUSIAUX et al. 2010a, b, ScHMULL et al. 2011), and, in many cases, has thoroughly reorganized the general phylogenetical topologies of higher taxa. Striking examples concern inter alia the Dothideomycetes (NELSEN et al. 2009), the Ostropales and especially the Graphidaceae and Thelotremataceae (BALOCH et al. 2010, Mangold et al. 2008, Rivas Plata \& Lumbsch 2011), the Arthoniales (ERTZ \& TEhLER 2011), and the Verrucariaceae (GUeIDAN et al. 2009). Finally we may be at the edge of a profund shift in the paradigm of using morphological and chemical characters to distinguish species within the lichenized fungi: indeed, several studies have demonstrated that no molecular support can be found for the classic handling of species delimitation in several common genera (LuMBSCH \& LEAVITT 2011).

The lack of comprehensive lichen checklists including recently assessed records is striking for many parts of the world (data and evaluation available at http://www.biologie.uni-hamburg.de/ checklists/lichens/portalpages/portalpage_checklists_switch.htm). Such lack of data precludes most phylogeographical analysis, albeit the few studies published so far have all yielded interesting and quite unexpected results (Printzen et al. 2003, WiRTZ et al. 2008, TeHLER et al. 2009, 2010, Hestmark et al. 2011, SÉrusiaux et al. 2011). We are thus convinced that providing correctly assessed lists of species occuring in a delimited area, even when incomplete, is urgently needed to allow for such comprehensive evaluation of $\alpha$-diversity throughout the world, in order to detect hotspots and study speciation and dispersion processes.

The Mascarene archipelago comprises three small volcanic islands located 640 to 1,450 km E of Madagascar: Mauritius (1,865 $\left.\mathrm{km}^{2}\right)$, Reunion $\left(2,512 \mathrm{~km}^{2}\right)$ and Rodrigues $\left(109 \mathrm{~km}^{2}\right)$. Their topography is very much influenced by their volcanic histories and duration of weathering since the last volcanic events (BuRGESs et al. 2004). Reunion (La Réunion in French) has one of the most active volcanoes in the world, the Piton de la Fournaise, reaching 2,631 m in elevation and responsible for a large and spectacular lava field on the eastern side of the island. Further, it is a serrated mountain range with the highest point at 3,069 $\mathrm{m}$ at the Piton des Neiges, dissected in huge “cirques" and very deep gorges. Its position in the tropical zone of the Indian Ocean (c. $21^{\circ} 05^{\prime} \mathrm{S} / 55^{\circ} 30^{\prime} \mathrm{E}$ ) makes its climate warm and seasonal, although the temperatures can get much lower at high elevation (mean temperature of c. $12^{\circ} \mathrm{C}$ at $2000 \mathrm{~m}$ ). A steep gradient in rainfall (from 10,000-12,000 mm at Hauts-de-Ste-Rose to $540 \mathrm{~mm}$ at StGilles) is a further characteristic of the island.

CADET (1977) distinguished three main forest types: the semi-arid forest ("forêt semi-sèche"), the lowland rainforest ("forêt humide de basse altitude"), and the mountain rainforest ("forêt humide de montagne") as well as other plant formations such as the highland rainforest ("forêt de moyenne altitude"). Semi-arid forests occur up to $750 \mathrm{~m}$ above sea level in areas with an annual precipitation lower than $1500 \mathrm{~mm}$ and a significant dry season; they form thickets of lower height, which develop into tree savannah with Latania lontorides and Terminalia bent- 
zoe in the driest areas of the west. Lowland rainforests are restricted to areas with an annual rainfall over $1500 \mathrm{~mm}$ per year. Characteristic trees are e.g. Doratoxylon apetalum, Molinea alternifolia, Labourdonnaisia callophylloides and Syzygium borbonicum. Above both forests types, at altitudes from $600-900 \mathrm{~m}$ in the east and 750-1100 $\mathrm{m}$ in the west, highland rainforests (forêt de moyenne altitude) with Badula grammistica, Cordemoya integrifolia and Euodia borbonica (DOUMENGE \& RENARD 1989) occur. Mountain rainforests climb up to $2000 \mathrm{~m}$ and are delimitated by the 11 and $15^{\circ} \mathrm{C}$ mean annual isotherms. The annual rainfall is usually higher than $2000 \mathrm{~mm}$ per year, with no dry season. Compared to lowland rainforests, the plant diversity of mountain forests is generally lower and the number of epiphytes higher. An early succession stage of mountain rainforests is the "forêt à Tamarins", characterised by the endemic tree Acacia heterophylla. Ericaceae, especially Erica montana, and Stoebe passerinoides dominate areas above 1600-1900 m (CADET 1977).

Humans colonized the Mascarene archipelago very late in history. Mauritius was discovered only in 1598, and colonization of the other two islands followed immediately (CHEKE \& HuME 2008). At that time the biomes of the three islands were untouched. As on other oceanic archipelagos and other "big" islands such as New Zealand and Madagascar, the impact has been dramatic and devastating. Island biota are characterized by a high level of endemism, active speciation and adaptative radiation, and demonstrate the relative importance of contingency and determinism in evolutionary diversification (Losos \& RicKLEFs 2009). They do not resist for long to human impacts (CHEKE \& Hume 2008), and current worldwide threats to the oceanic islands biodiversity are unprecedented (CAUJAPÉ-CASTELls et al. 2010). Well-known extinctions in the Mascarene archipelago include two giant flightless birds, the dodo (Raphus cucculatus) on Mauritius and the solitaire (Pezophaps solitaria) on Rodrigues, formerly considered to form an entire family (the Raphidae) but now included within the Columbidae (SHAPIRo et al. 2002). Furthermore, no less than five species of giant tortoises in the genus Cylindraspis (GERLACH 2004) have become extinct. Low elevation vegetation has been almost eradicated on all islands, with very few exceptions such as Round Island, a 169 ha islet north of Mauritius which harbors the last palm-savanna stand in the whole archipelago, with extreme rare palms such as Latania loddigesii, Dictyosperma album var. conjugatum and Hyophorbe lagenicaulis, the latter two being now confined to the islet (NoRTH et al. 1994).

Nevertheless, large segments of the biodiversity of the Mascarene archipelago and their phylogeography remain to be studied: new endemic species continue to be described, such as in the Orchidaceae and Selaginella (Bosser 2007, Delmail 2009).

Two islands of the Mascarene archipelago (Mauritius and Reunion) are famous in the literature in terms of lichen taxonomy. Indeed, many types of characteristic and/or widespread species originate from either of these two islands. This is the result of the collections made in 1801 by J. B. G. M. Bory de Saint-Vincent (1778-1846), a French officer who joined a scientific expedition in 1800 on the ship « Le Naturaliste ». He visited several islands around Africa, including Tenerife in the Canary Islands, St Helena, and the Mascarene archipelago, landing on Mauritius in March 1801, and on Reunion in May 1801. He climbed the Piton de la Fournaise, the most famous and active volcano of the island, and made splendid and copious collections of lichens on both islands (LACROIX 1917, SAuvagEAu 1908). In 1804, he published the threevolume «Voyage dans les quatre principales îles d'Afrique» which contains accounts of the lichens he collected on Mauritius and Reunion. He described several well-known and spectacular species: Lichen candelabrum (=Cladonia candelabrum), Lichen giganteus (=Cladonia gigantea), Lichen medusinus (=Cladonia medusina), Lichen unguigerus (=Erioderma unguigerum), Lichen retiger (=Lobaria retigera), Lichen salazinus (=Stereocaulon salazinum), 
Lichen vulcanus (=Stereocaulon vulcani), Lichen ambavillarius (=Sticta ambavillaria) and Lichen variabilis (= Sticta variabilis). His material was further examined by leading lichenologists such D. F. Delise, who described additional new taxa (Delise 1825): Leioderma erythrocarpum (Delise ex Nyl.) D.Galloway \& P.M.Jørg., Lobaria discolor (Delise) Hue and Pseudocyphellaria argyracea (Delise) Vain.

Several papers made a significant contribution to the knowledge of lichens in the Mascarene archipelago, such as those by NyLANDER (1859), Des Abbayes (1961) and Mathey (1971). More recently, this lichen flora has been brought back into attention by contributions to the genera Pannaria by Jørgensen (2004), Parmeliella by JøRGEnSEN \& SCHumm (2010), Phyllopsora by Timdal \& Krog (2001) and Solenopsora by Verdon \& RAMBold (1998), and on representatives of the Arthoniales (ERTZ \& TeHLER 2011). Two floristic contributions were dedicated to the foliicolous lichen flora (SÉrusiaux 1977, RonHEDE et al. 2003), and an expertise report has been released on the quality of air around the island, based on the epiphytic lichen flora (LEROND 1998). In spite of this, the lichen flora of Reunion is poorly known and has never been thoroughly revised. A preliminary checklist is available at http://www.biologie. uni-hamburg.de/checklists/lichens/africa/france_reunion_l.htm.

A website with identification keys is found at http://www.bgbm.fu-berlin.de/sipman/Zschackia/ Reunion/Cladonia.htm. Currently it contains the genera Cladonia (illustrated), Erioderma, Porina and Pyrenula.

The lichen flora of the island is currently under investigation by our group. We here provide a first contribution to the knowledge of the lichens diversity on the island, based only on our own collections gathered during field trips starting in 1991, and furthermore on examination of collections preserved in B (collections made by G. Follmann in 1989) and LG (collections made by J. L. de Sloover in 1973).

\section{Material and methods}

About 2500 specimens of lichens were investigated with a light-microscope and/or binocular microscope, and partly by TLC according to ORANGE et al. (2001). Several specimens were checked or identified by specialists: Ted Ahti (Cladonia), Jack Elix (Parmeliaceae), Mireia Giralt (Rinodina and related genera), Per Magnus Jørgensen (Pannariaceae) and Roland Moberg (Physcia).

Published results from recent work on our collections, including the description of new species, are not repeated here. These include 29 species:

Alyxoria varia (Pers.) Ertz \& Tehler s.l. (=Opegrapha varia Pers.) (ERTz 2009)

Arthonia borbonica Ertz, Elix \& Grube (ERTz et al. 2010)

Bacidiopsora microphyllina Kalb (KALB 2004)

Bapalmuia cacaotica Kalb \& Lücking (KALB 2004)

Brigantiaea substipitata Kalb \& Hafellner (HAFELLNER 1997)

Conotremopsis weberiana Vězda (KaLB 2004)

Crypthonia palaeotropica A.Frisch \& G.Thor and C. vandenboomii A.Frisch \& G.Thor (Frisch \& THOR 2009)

Erioderma borbonicum P.M.Jørg. \& van den Boom, E. papyraceum P.M.Jørg. \& Arv., E. sorediatum P.M.Jørg. \& D.J.Galloway, and E. unguigerum (Borrer) Nyl. (JøRGENSEN et al. 2009)

Fellhanera borbonica Sérus., van den Boom \& M.Brand, (Lumbsch et al. 2011)

Gassicurtia nordinii Kalb \& Elix (KaLB et al. 2009b)

Lecanactis borbonica Ertz \& Tehler (ERTZ \& TEHLER 2011) 
Melanotopelia africana Sérus., M.Brand, Ertz, Fischer, Killmann \& van den Boom (SÉRUSIAUX et al. 2009) Opegrapha agelaeotera Vain. and Opegrapha borbonica Ertz (ERTz 2009)

Opegrapha viridistellata Sérus., Lücking \& Sparrius (ERTZ \& TeHLER 2011)

Parmeliella borbonica P.M.Jørg.\& Schumm (JørgenSEN \& Schumm 2010)

Sculptolumina japonica (Tuck.) Marbach (GIRALT et al. 2009)

Sipmaniella sulfureofusca (Fée) Kalb (KALB et al. 2009a)

Syncesia glyphysoides (Fée) Tehler, S. madagascariensis Ertz, Killmann, Razafin., Sérus. \& Eb.Fisch.

and S. mascarena van den Boom, Ertz, M.Brand \& Sérus. (van Den Boom et al. 2011)

Thelotrema reunionis Kalb (KALB 2009)

Tephromela gigantea Kalb \& Elix (KALB 2004)

Vainionora aemulans (Vain.) Kalb (KALB 2004)

Zwackhia involuta (Wallr.) Körb. [= Opegrapha viridis (Ach.) Behlen \& Desberger] (ERTz 2009).

Further, a single collection by one of us (KK) has been issued in Exsiccati: Baculifera intermedioides Marbach (K. Kalb: Lichenes Neotropici, fasc. 14, $n^{\circ} 581$ ).

Localities where collections have been made can be identified on the map (Fig. 1). Local ecology of the species is briefly mentioned, usually as corticolous, saxicolous, terricolous or foliicolous. Collections are preserved in B (collections by G. Follmann and U. Schiefelbein, abbreviated as GF and US, respectively), BR (collections by D. Ertz, abbreviated as DE), and LG (collections by N. Magain, J. L. de Sloover and E. Sérusiaux), in the Lichenologisches Institut Neumarkt (collections by K. Kalb, abbreviated as KK), as well as in the private herbaria of M. Brand (abbreviated as MB), D. Masson (abbreviated as DM) and P. van den Boom (abbreviated as PB). Only a few representative collections for each reported species are mentioned in this paper. It is beyond the aim of this preliminary list to assess the abundance of each species. A set of representative collections will be deposited at the Herbarium of the Reunion University in St-Denis (REU).

\section{Results and discussion}

434 lichen taxa (species and infraspecific taxa) are recognized in our material and are documented in this paper. In the list below, most records are from the Cirque de Cilaos (210), the Forêt de Bébour (155), the Plaine des Palmistes (99) and the Piton de la Fournaise (97). The list below is not an authentic checklist as many published reports have not been checked and many more specimens remain to be identified, even macrolichens or supposedly well-known taxa. As large parts of our collections are still unnamed, it can be expected that the actual lichen flora of Reunion exceeds 600 species. This is probably a rather low figure for a tropical region, since limited forest plots are known to contain c. 250 species in continental hotspots (Komposch \& HAFEllner 1999). A reduced diversity is to be expected for remote and small islands like Reunion. For example, 376 species have been reported from the Seychelles archipelago (SEAWARD \& APTROOT 2009).

The significance of Reunion for lichen biodiversity is more likely in terms of endemic taxa, strictly limited to the island, to the Mascarene archipelago, or further to Madagascar and the Mascarene archipelago. Although the lichen flora of this region is poorly known, especially in Madagascar, species strictly endemic to Reunion seem to occur, such as the conspicuous and easily recognized species Cladonia insignis Nyl. (Stenroos 1991), Cl. mascarena Nyl. (Ahti \& Aptroot 1992) and Stereocaulon salazinum (Bory) Fée (LAMB 1977). However, other recently described or recognized species so far thought to be endemic to Reunion are likely to be discovered elsewhere, especially if more research is conducted in countries surrounding the 


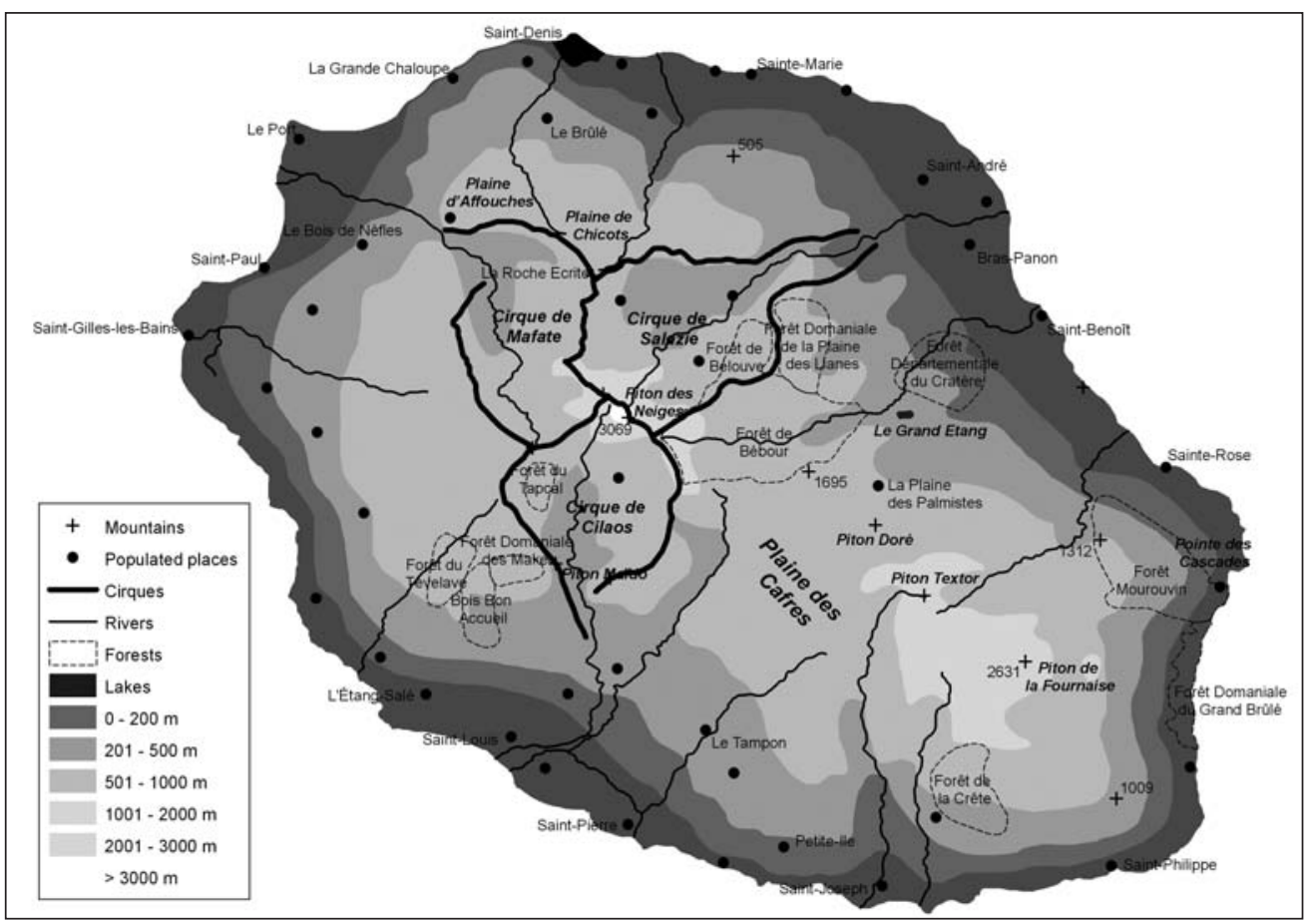

Fig. 1: Map of the island of Reunion (Mascarene archipelago) with main cities and all collecting localities mentioned in this paper. Prepared by Ulf Schiefelbein.

Indian Ocean (Madagascar, eastern coasts of Africa, India). We nevertheless think that endemism is likely to be underestimated. Indeed, identified taxa usually belong to taxonomically "better-known" groups and well represented in monographs and floristic treatments, while endemic taxa are likely to be left unidentified because of complicated and understudied taxonomies. For a careful assessment of the endemic element, regional monographic studies will be required for most lichen groups.

A further point must be made for the unexpected detection of an austral element, that is species likely to be confined to the southern hemisphere (GALLOWAY 2008). No phylogeographical studies have ever been conducted for those taxa and thus no strong evidence can be provided on their origin: either paleoaustral as being “primitive Gondwanan” groups or neoaustral as having being dispersed throughout the southern hemisphere after the fragmentation of the Gondwana, or originating from the northern hemisphere and having been dispersed to the South where speciation occurred. Nevertheless, several discoveries made on Reunion are remarkable: Peltigera sorediifera, furthermore known from continental Africa south of the equator and Australia/Queensland, Gomphillus morchelloides, formerly known from Papua New Guinea and Chile (LÜCKING \& SÉrusiaux 2005), a further species from the same genus, $G$. pedersenii formerly known only from its type locality in Argentina (FERRARO \& LÜCKING 2005), Lobaria asperula (Stirt.) Yoshim., formerly known from New Zealand and a single locality in eastern Tanzania (GAlloway 2007, Krog 2000), the very characteristic Sporopodiopsis mortimeriana, so far only known from Papua New Guinea (SÉrusiaux 1997) and Lithogyalideopsis zeylandica, formerly known only from New 

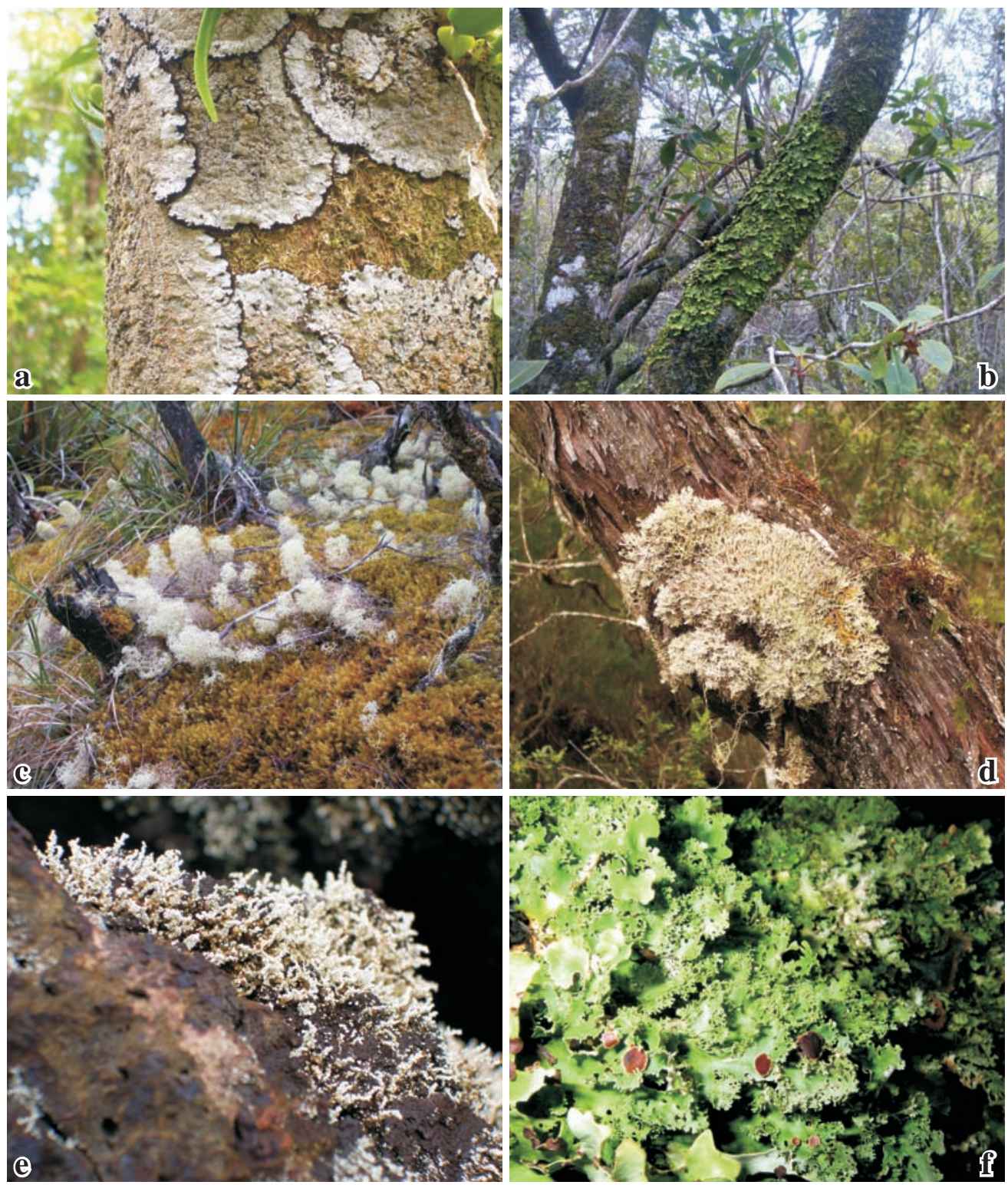

Fig. 2: Photographs of several conspicuous species present on Reunion island. a - Parmeliella mariana near StPhilippe, b - Sticta caperata in Cirque de Cilaos, c - Cladonia confusa in Forêt de Bébour, d - Siphula mascarena in Forêt de Bébour, e - Stereocaulon vulcani at Piton de la Fournaise, $\mathbf{f}$ - Sticta variabilis in Forêt de Bébour. Photographs taken in the field by Emmanuël Sérusiaux and Bern van den Boom.

Zealand (VĚZdA \& Malcolm 1997). The discovery of those species in Reunion is clearly the result of the expertise of our team as it is remarkable that none of these have ever been detected elsewhere in tropical regions where we have assembled large and representative lichen collections. We thus speculate that several representatives of a genuine austral lichen element managed to reach and flourish on Reunion. 


\section{Species list}

Aderkomyces albostrigosa (R.Sant.) Lücking, Sérus. \& Vězda - Grand Étang PB40071. Foliicolous.

Aderkomyces armatus (Vězda) Lücking, Sérus. \& Vězda - Cirque de Cilaos LG. Foliicolous.

Agonimia opuntiella (Buschardt \& Poelt) Vězda - Cirque de Cilaos PB41040; Le Tampon PB40849. Saxicolous.

Agonimia pacifica (Harada) Diederich - Forêt Domaniale du Grand Brûlé KK33564. Corticolous.

Agonimia tristicula (Ach.) Zahlbr. - Piton de la Fournaise PB40635. Lignicolous.

Amandinea placodiomorpha (Vain.) Marbach St-Benoît PB40995; St-Philippe PB40175. Corticolous.

Ancistrosporella curvata (Aptroot) Komposch - Plaine des Palmistes PB39778, LG, MB. Corticolous.

Anthracothecium macrosporum (Hepp) Müll. Arg. - Cirque de Cilaos US1461, KK33692. Corticolous.

Anzia afromontana R.Sant. - Cirque de Cilaos KK26440. Corticolous. As most species of Anzia, A. afromontana most probably belongs to the austral elements: it is known from East Africa, Papua New Guinea and South America (Yoshimura et al. 1995, Calvelo 1996).

Anzia corallophora Yoshim. - Cirque de Salazie KK33496; Forêt de Bébour LG. Corticolous. Formerly known only from Papua New Guinea (Yoshimura et al. 1995).

Arthonia cinnabarina (DC.) Wallr. - Plaine des Palmistes PB39806, LG. Corticolous.

Arthonia speciosa (Müll.Arg.) Grube - Cirque de Cilaos US1637. Corticolous.

Arthrorhaphis citrinella (Ach.) Poelt - Cirque de Cilaos US1616. Terricolous.

Bacidina apiahica (Müll.Arg.) Vězda - Cirque de Salazie PB40929. Foliicolous.

Bacidiopsora squamulosula (Nyl.) Kalb - Cirque de Salazie PB40968; Forêt de Bébour PB40336; Plaine des Palmistes PB39891. Corticolous.

Badimia pallidula (Kremp.) Vězda - Forêt de Bébour LG. Foliicolous on filmy fern.

Baeomyces rufus (Huds.) Rebent. - Piton de la Fournaise PB39961. Terricolous.

Bapalmuia halleana Sérus. - Grand Étang LG; Plaine des Palmistes PB39736; St-Philippe PB40582. Over corticolous mosses.
Brigantiaea leucoxantha (Spreng.) R.Sant. \& Hafellner - Cirque de Cilaos PB40259, LG, US1460, KK25181; Grand Étang KK25527; Plaine d'Affouches KK26002; Plaine des Palmistes PB39896, DE4083; St-Benoît PB41030, LG. Corticolous.

Brigantiaea tricolor (Mont.) Trevis. - Cirque de Cilaos US1590, KK26162; Forêt de Bébour LG; Plaine d'Affouches KK26373; between StBenoit and Ste-Rose KK26143. Corticolous.

Buellia ceylonensis Zahlbr. - Cirque de Salazie KK26462. Saxicolous.

Buellia bahiana Malme - St-Philippe PB40162. Corticolous.

Buellia parastata (Nyl.) Zahlbr. - Grand Étang PB40076; Le Tampon PB40796; Piton Doré to Piton Textor KK33582; St-André PB40697; Ste-Rose PB40149. Corticolous.

Bulbothrix goebelii (Zenker) Hale - Forêt de Bébour PB40422. Corticolous.

Bulbothrix suffixa (Stirt.) Hale - Plaine des Palmistes PB39841. Corticolous.

Bunodophoron melanocarpum (Sw.) Wedin Cirque de Cilaos US1634; Forêt de Bébour PB40363, LG. Corticolous.

Byssoloma leucoblepharum (Nyl.) Vain. - Cirque de Salazie PB409632; Plaine des Palmistes PB39817. Corticolous or foliicolous.

Byssoloma marginatum (Arnold) Sérus. - Forêt de Bébour LG; Plaine des Palmistes PB39793. Corticolous.

Byssoloma subdiscordans (Nyl.) P.James - Cirque de Cilaos US1552; Cirque de Salazie PB40962; Plaine des Palmistes PB39816. Corticolous or foliicolous.

Calicium hyperelloides Nyl. - Cirque de Cilaos US1450; Piton de la Fournaise PB40003, LG; between St-Denis and Plaine des Chicots DE4801. Corticolous.

Calopadia phyllogena (Müll.Arg.) Vězda - Cirque de Salazie PB40925; Plaine des Palmistes PB40916. Foliicolous.

Caloplaca caesiosorediata Arup \& van den Boom - Cirque de Salazie PB40732; St-Benoît PB40743; St-Philippe PB40164. Corticolous. Species recently described from the Cape Verde Archipelago in the Atlantic Ocean (Arup \& VAN DEN Boom 2011).

Caloplaca chrysodeta (Vain. ex Räsänen) Dombr. - St-Benoît PB41019. Saxicolous.

Caloplaca kurzii (Kremp.) Zahlbr. - Cirque de Cilaos KK25203. Saxicolous. 
Canoparmelia aptata (Kremp.) Elix \& Hale - Cirque de Mafate DM974.1787; St-Paul DM974.0251. Corticolous.

Canoparmelia texana (Tuck.) Elix \& Hale - StPaul, MB59499. Corticolous.

Catinaria atropurpurea (Schaer.) Vězda \& Poelt - Cirque de Cilaos KK26013; Piton Doré to Piton Textor KK33592; Plaine des Palmistes PB39899. Corticolous.

Celothelium aciculiferum (Nyl.) Vain. - Forêt de Bébour DE4292b; St-Philippe DE4396. Corticolous.

Chaenotheca brunneola (Ach.) Müll.Arg. Cirque de Cilaos US1530. Corticolous.

Chaenotheca furfuracea (L.) Tibell - Cirque de Cilaos US1558. Corticolous.

Chiodecton leptosporum Müll.Arg. - Forêt de Bébour MB58816, KK25493, DE4078; Forêt de Bélouve KK33695; Le Tampon PB40748; between St-Benoit and Ste-Rose KK26383; StPaul MB59477; between St-Denis and Plaine des Chicots DE4776. Corticolous.

Chrysothrix xanthina (Vain.) Kalb - Cirque de Cilaos PB40227; St-Benoît PB40739. Corticolous.

Cladia aggregata (Sw.) Nyl. - Cirque de Cilaos US1574; SE of Piton des Neiges DE4692 ; Forêt de Bébour PB39959; Piton de la Fournaise PB39959, GF (B 60 0149596). Terricolous.

Cladonia andesita Vain. - Cirque de Cilaos US1607; Piton de la Fournaise GF (B 60 0149667). Terricolous.

Cladonia boivinii Vain. (= Cl. poeciloclada Abbayes) - Cirque de Cilaos KK26273; Forêt de Bébour MB58834; Piton de la Fournaise GF (B 60 0149668), LG; Piton des Neiges MB59859. Terricolous.

Cladonia borbonica Nyl. - Cirque de Cilaos KK26302; Piton de la Fournaise GF (B 60 0135155); Plaine des Palmistes MB59302; StAndré MB59108. Terricolous.

Cladonia candelabrum (Bory) Nyl. - Cirque de Cilaos DE4704, MB59821, US1608; Forêt de Bébour PB 40493, MB58912, LG; Piton de la Fournaise GF (B 60 0135133). Terricolous.

Cladonia centrophora Müll.Arg. - Cirque de Cilaos US1698, MB59658; Forêt de Bébour PB40320, MB58748, LG; Le Tampon MB59181; Piton de la Fournaise GF (B 60 0135089), MB59597, LG. Terricolous.

Cladonia cinereorubens Abbayes - Grand Étang PB40093; Plaine des Palmistes MB58488. Terricolous.
Cladonia coccifera (L.) Willd. - Cirque de Cilaos US1691. Terricolous.

Cladonia confusa (Sandst.) Follmann \& Ahti - Cirque de Cilaos MB59820, US1619, LG, KK33686; Forêt de Bébour PB40421, MB58835, LG. Terricolous.

Cladonia didyma (Fée) Vain. - Cirque de Cilaos MB59772, US1593, KK33755; Cirque de Salazie KK33732; Forêt de Bébour MB58745, PB40345, LG; Piton de la Fournaise MB58338; Plaine des Palmistes PB39877. Corticolous and terricolous.

Cladonia fruticulosa Kremp. - Cirque de Cilaos KK25957; Forêt de Bébour MB58747; SteRose MB58570. Humicolous.

Cladonia gigantea (Bory) H.Olivier - Cirque de Cilaos US1621; Forêt de Bébour PB40417, MB58831, LG. Terricolous.

Cladonia glaucopallida Vain. - Cirque de Cilaos US1557; Forêt de Bébour MB58832. Terricolous.

Cladonia hedbergii Ahti - Cirque de Cilaos US1595; Piton de la Fournaise GF (B 60 0135138). Terricolous.

Cladonia insignis Nyl. - Cirque de Cilaos DE4686, US1603; Forêt de Bébour PB40535, MB58838, LG. Terricolous.

Cladonia intermediella Vain. - Cirque de Cilaos US1680, KK25179; Piton de la Fournaise GF (B 60 0135154), MB59596. Terricolous.

Cladonia leucophylla Ahti \& Krog - Cirque de Cilaos DE4101, US1668, MB59915; Forêt de Bébour PB39751, MB59298. Corticolous and terricolous.

Cladonia macilenta Hoffm. - Cirque de Cilaos KK33690; Forêt de Bébour MB58178; Petite-Ile LG; Piton de la Fournaise GF (B 60 0135156); Plaine des Palmistes PB40805. Corticolous.

Cladonia mascarena Nyl. - Forêt de Bébour PB40515, LG, MB58836; St-Paul MB59533. Terricolous.

Cladonia mauritiana Ahti \& J.C.David - Cirque de Cilaos US1436, MB59625; Cirque de Salazie KK33492; Forêt de Bébour MB59299; Piton de la Fournaise PB40842, GF (B 60 0135099, B 60 0135098); Plaine des Palmistes MB58144; St-André MB59108; Ste-Rose PB40182, MB58569; St-Philippe GF (B 60 0135434), MB58967. Terricolous.

Cladonia medusina (Bory) Nyl. - Cirque de Cilaos US1689; Forêt de Bébour PB 40317, MB58842; Piton de la Fournaise PB39956, LG; Plaine des Palmistes PB40912, LG; St-André PB40727. Corticolous and terricolous. 
Cladonia ochrochlora Flörke - Piton de la Fournaise PB40007, GF (B 60 0135183). Corticolous.

Cladonia pachyclados (Vain.) Ahti - Forêt de Bébour, LG; Piton de la Fournaise GF (B 60 0128914); St-Philippe GF (B 60 0135449). Terricolous.

Cladonia peltasta (Ach.) Spreng. - Cirque de Cilaos MB59819, US1512; Forêt de Bébour PB40397, MB58842, LG; Piton de la Fournaise MB58283, GF (B 60 0135131); Piton des Neiges MB59861; Plaine des Palmistes MB59301; StPaul MB59531. Terricolous.

Cladonia perrieri Abbayes - Cirque de Cilaos MB59822, US1591; Forêt de Bébour PB40543, MB58831, LG. Terricolous.

Cladonia peziziformis (With.) J.R.Laundon Piton de la Fournaise PB39962; Plaine des Chicots KK33428. Terricolous.

Cladonia squamosa Hoffm. - Cirque de Cilaos US1700, KK33754; Forêt de Bébour PB40418, MB58918, LG; Piton de la Fournaise PB39954; Plaine des Palmistes PB39859, MB59300; Ste-Rose MB58569. Corticolous and terricolous.

Cladonia subdelicatula Vain. ex Asah. - Forêt de Bébour PB40347; Grand Étang PB40089, MB58490. Corticolous. Confirmed by T. Ahti. This species, formerly known from South America (Анті 2000), might be common in tropical Africa.

Cladonia submultiformis Asah. - Cirque de Cilaos US1510; Piton de la Fournaise GF (B 60 0135152). Terricolous.

Cladonia subsquamosa Kremp. - Cirque de Cilaos US1592, MB59757; Forêt de Bébour MB58841; Grand Étang MB58490; Piton de la Fournaise GF (B 60 0135097). Corticolous.

Cladonia varians Vain. ex Ahti - Cirque de Cilaos US1582; Forêt de Bébour MB58839; Piton de la Fournaise LG; Piton des Neiges MB59859. Terricolous.

Coccocarpia delicatula Bungartz, Ziemmeck \& Lücking - Forêt de Bébour PB40522, LG. Corticolous. A species recently described from the Galapagos Archipelago (Lumbsch et al. 2011).

Coccocarpia erythroxyli (Spreng.) Swinscow \& Krog - Forêt de Bébour PB40452, MB5889; StPhilippe DM974.0179. Corticolous.

Coccocarpia filiformis Arv. - Forêt de Bébour PB39929; St-Philippe PB40581. Corticolous.
Coccocarpia palmicola (Spreng.) Arv. \& D.J.Galloway - Cirque de Cilaos US1424; Cirque de Salazie DM974.0331, KK25475; Forêt de Bébour DM974.0105, DE4079b; Grand Étang PB40042; Plaine des Palmistes PB39766; Ste-Marie DM974.0462 ; St-Philippe DM974.0177; Ste-Rose PB40154. Corticolous.

Coccocarpia pellita (Ach.) Müll.Arg. - Forêt de Bébour DM974.0108; St-André PB40711. Corticolous.

Coccocarpia pruinosa Arv. - Cirque de Cilaos US1710; Forêt de Bébour DM974.0426, KK25501; Grand Étang LG, KK25526; Plaine des Palmistes KK26499; Ste-Marie DM974.0456. Corticolous.

Coccocarpia smaragdina Pers. - Cirque de Cilaos US1533; Forêt de Bébour DM974.0365; Grand Étang PB40085; Le Tampon DM974.1382; Piton de la Fournaise GF (B 60 0135437); StPhilippe PB40060, LG, GF (B 60 0135447); Ste-Rose PB40114; KK33647. Corticolous.

Coccocarpia stellata Tuck. - Forêt de Bébour DE4292a, PB40360, LG. Corticolous.

Coccotrema porinopsis (Nyl.) Imshaug ex Yoshim. - Cirque de Cilaos PB40222; Forêt de Bébour PB40399, KK25216; Plaine des Palmistes PB39756. Corticolous.

Cratiria aggrediens (Stirt.) Marbach - Cirque de Cilaos US1630, KK33453. Corticolous.

Cratiria dissimilis (Nyl.) Marbach - Ste-Rose PB40148. Corticolous.

Cratiria obscurior (Stirt.) Marbach \& Kalb - Cirque de Salazie PB40733; St-Joseph PB40594. Corticolous.

Cresponea proximata (Nyl.) Egea \& Torrente Forêt de Bébour PB40873, LG. Corticolous.

Crocynia gossypina (Sw.) A.Massal. - St-Philippe PB40561, LG, DE4408. Corticolous.

Dibaeis holstii (Müll.Arg.) Kalb \& Gierl Cirque de Cilaos US1600; Forêt de Bébour PB40532; Forêt de Bélouve KK33381; Piton de la Fournaise GF (B 60 0135124); Plaine d'Affouches KK 33455; Plaine des Palmistes PB40442. Terricolous.

Dibaeis sorediata Kalb \& Gierl - La Roche Ecrite LG; S of Saint-Denis DE4765. Terricolous.

Dictyographa arabica Müll.Arg. - St-Philippe DE4211. Corticolous.

Dictyonema sericeum (Sw.) Berk. - Forêt de Bébour PB39933, LG; St-Philippe PB40588. Over corticolous mosses. 
Dictyonema sericeum f. schenkianum (Müll. Arg.) Parm. - Between St-Benoit and Ste-Rose KK33475. Over corticolous mosses.

Diorygma hieroglyphicum (Pers.) Staiger \& Kalb - Le Tampon PB40861. Corticolous.

Diploschistes actinostomus (Pers. ex Ach.) Zahlbr. - Cirque de Cilaos PB40219. Saxicolous.

Diploschistes euganeus (A.Massal.) J.Steiner - Le Tampon PB40860. Saxicolous.

Diploschistes hypoleucus (Vain.) Zahlbr. Between St-Benoit and Ste-Rose KK25643. Muscicolous.

Diploschistes muscorum (Scop.) R.Sant. subsp. bartlettii Lumbsch - Cirque de Cilaos US1573; Forêt de Bébour PB40548. Terricolous.

Diploschistes scruposus (Schreb.) Norman - Forêt de Bébour PB40457; Piton de la Fournaise PB39967. Saxicolous.

Dirinaria aegialita (Ach.) Moore - Cirque de Cilaos US1437, KK33748; Piton de la Fournaise GF (B 60 0135424); Ste-Rose DM974.0289. Corticolous.

Dirinaria applanata (Fée) D.D.Awasthi - Piton de la Fournaise GF (B 60 0135426); Piton Maïdo KK26392; St-Paul DM974.1926; between St-Benoit and Ste-Rose KK33610; Ste-Rose PB40139. Corticolous.

Dirinaria confluens (Fr.) D.D.Awasthi - St-Gillesles-Bains KK26517. Saxicolous.

Dirinaria picta (Sw.) Clem. \& Shear - Cirque de Cilaos US1541; St-Benoît PB40744. Corticolous.

Dyplolabia afzelii (Ach.) A.Massal. - St-Philippe PB40160; Forêt Domaniale du Grand Brûlé DE4240. Corticolous.

Echinoplaca pellicula (Müll.Arg.) R.Sant. Cirque de Cilaos, LG. Foliicolous.

Endocarpon johnstonii (Müll.Arg.) Stizenb. - Cirque de Salazie PB40984; St-Joseph PB40599. Saxicolous.

Enterographa anguinella (Nyl.) Redinger Le Tampon MB59232; Ste-Rose PB40115. Corticolous.

Enterographa multiseptata R.Sant. - Ste-Rose PB40138, LG. Foliicolous.

Enterographa pallidella (Nyl.) Redinger - StJoseph MB58975; St-Philippe DE4212. Corticolous.

Enterographa tropica Sparrius - St-Philippe DE4213. Corticolous.

Enterographa zonata (Körb.) Torrente \& Egea Forêt de Bébour PB40475. Saxicolous.
Ephebe lanata (L.) Vain. - Forêt de Bébour KK33616. Saxicolous.

Ephebe ocellata Henssen - Forêt de Bébour MB58315; Piton de la Fournaise MB58432; StAndré MB59097. Saxicolous.

Erioderma groendalianum (Ach.) Vain. - Cirque de Cilaos LG, KK25955; Piton de la Fournaise GF (B 60 0142002). Corticolous.

Erioderma leylandii (Tayl.) Müll.Arg. - Cirque de Salazie KK33738. Piton des Neiges MB59789. Corticolous.

Eschatogonia marivelensis (Vain.) Kalb - Forêt de Bébour PB40388; Plaine des Palmistes PB39731. Corticolous.

Fellhanera bouteillei (Desm.) Vězda - Cirque de Salazie PB40928; Grand Étang PB40072. Foliicolous.

Fibrillithecis argentea (Müll.Arg.) Rivas Plata \& Lücking - Between St-Benoit and SteRose KK25962; Cirque de Cilaos KK25491. Corticolous.

Fibrillithecis insignis (Zahlbr.) A.Frisch - Forêt de Bébour PB40377, LG. Corticolous.

Fibrillithecis vernicosa (Zahlbr.) A.Frisch - Forêt de Bébour PB40432, LG; Plaine des Palmistes PB39826. Corticolous.

Fissurina comparilis (Nyl.) Nyl. - Cirque de Cilaos PB40248; Forêt de Bébour PB40486; Grand Étang PB40099; Plaine des Palmistes PB39813. Corticolous.

Fissurina columbina (Tuck.) Staiger - Forêt de Bébour DE4154. Corticolous.

Fissurina dumastii Fée - St-Philippe DE4347. Corticolous.

Fissurina triticea (Nyl.) Staiger - Le Tampon PB40769; Piton de la Fournaise PB40667. Corticolous.

Flakea papillata O.E.Erikss. - Forêt de Bébour PB40357, LG; Le Tampon PB40836; St-Benoît PB40996; St-Joseph PB40609. Corticolous and saxicolous.

Fuscopannaria leucosticta (Tuck.) P.M.Jørg.Forêt de Bébour PB40519, LG. Corticolous.

Fuscopannaria praetermissa (Nyl.) P.M.Jørg.Cirque de Cilaos LG. Terricolous. A remarkable record for this widespread species, that is usually considered as a typical representative of the "arctic-alpine” element (JøRGENSEN 2003). It also occurs on several of the highest summits of East Africa (Mt Kenya and Karisimbi in the Virunga range), as well as on a high summit in Ecuador, South America (Jørgensen \& PAlice 2010). 
Gassicurtia elizae (Tuck.) Marbach - Between StBenoit and Ste-Rose KK333374. Corticolous.

Gassicurtia ferruginascens (Malme) Marbach \& Kalb - Le Tampon PB40803. Corticolous.

Gassicurtia vaccinii (Vain.) Marbach, Elix \& Kalb - Cirque de Cilaos KK33760; Forêt de Bébour PB39792; Le Tampon PB40787; Piton Doré to Piton Textor KK33758. Corticolous.

Glyphis cicatricosa Ach. - Cirque de Cilaos PB40274; Plaine des Palmistes PB39807; StAndré PB40699; St-Philippe PB40168; SteRose PB40135. Corticolous.

Gomphillus morchelloides Lücking \& Sérus. Forêt de Bébour PB40499, LG. Over corticolous mosses. Species previously known only from its type locality in Papua New Guinea and a second locality in Chile (LüCKING \& SÉrUSIAUX 2005).

Gomphillus pedersenii L.I.Ferraro \& Lücking - Forêt de Bébour LG; St-André PB40716. Over corticolous mosses. Species previously known only from its type locality in Argentina (FerRaro \& LüCKING 2005).

Graphis acharii Fée - Cirque de Cilaos US1653; Plaine des Palmistes PB39886; between St-Denis and Plaine des Chicots DE4812. Bambusicolous and corticolous.

Graphis antillarum Vain. - Cirque de Salazie PB40722. Corticolous.

Graphis argentata Lücking \& L.Umaña - Cirque de Cilaos PB40272; Grand Étang PB40102; Plaine des Palmistes PB39788. Corticolous.

Graphis aurita Eschw. - St-Philippe PB40591. Corticolous.

Graphis bettinae Lücking, L.Umaña, Chaves \& Sipman - Cirque de Cilaos PB40273. Corticolous.

Graphis calcea (Fée) A.Massal. - Le Tampon PB40795. Corticolous.

Graphis chlorotica A.Massal. - Plaine des Palmistes PB39912. Corticolous.

Graphis cincta (Pers.) Aptroot - Plaine des Palmistes PB39802. Corticolous.

Graphis conferta Zenker - Piton de la Fournaise PB39972. Corticolous.

Graphis crebra Vain. - Le Tampon PB40831. Corticolous.

Graphis dolichographa Nyl. - Cirque de Salazie PB40990. Corticolous.

Graphis emersa Müll.Arg. - Between St-Denis and Plaine des Chicots DE4802. Corticolous.

Graphis furcata Fée - Cirque de Cilaos US1472; Plaine des Palmistes PB39815; St-Philippe DE4414. Corticolous.
Graphis granulocarpa Redinger - Cirque de Cilaos US1651. Corticolous.

Graphis handelii Zahlbr. - Cirque de Cilaos DE4668, US1467. Corticolous.

Graphis leptoclada Müll.Arg. - Forêt de Bébour PB40351. Corticolous.

Graphis myrtacea (Müll.Arg.) Lücking - Le Tampon PB40812. Corticolous.

Graphis pinicola Zahlbr. - Cirque de Cilaos US1441. Corticolous.

Graphis proserpens Vain. - Cirque de Cilaos DE4677; Forêt de Bébour PB40376; Grand Étang PB40100; Le Tampon PB40767; Piton de la Fournaise PB39973; Plaine des Palmistes PB39722; St-André PB40724; Cirque de Cilaos DE4677. Corticolous.

Graphis rhizocola (Fée) Lücking \& Chaves Cirque de Cilaos PB40253; Plaine des Palmistes PB40618. Corticolous.

Graphis striatula (Ach.) Spreng. - Plaine des Palmistes PB39780. Corticolous.

Graphis subserpentina Nyl. - Grand Étang PB40064; St-Benoît PB41018. Corticolous and saxicolous.

Graphis submarginata Lücking - Cirque de Cilaos US1441. Corticolous.

Graphis tenella Ach. - Grand Étang PB40101. Corticolous.

Graphis vittata Müll.Arg. - Cirque de Cilaos US1646. Corticolous.

Gyalidea hyalinescens (Nyl.) Vězda - Cirque de Cilaos US1554. Terricolous.

Gyalideopsis bispora Vězda - Forêt de Bébour PB40451, LG; Piton de la Fournaise PB39978. Corticolous. A species so far only known as foliicolous in Tanzania (VĚZDA 1979).

Haematomma africanum (J.Steiner) C.W.Dodge Piton Maïdo KK33479. Corticolous.

Haematomma collatum (Stirt.) C.W.Dodge Cirque de Cilaos PB40199, US1537, KK26026; Cirque de Salazie PB40989; Le Tampon PB40779; Plaine des Palmistes PB39804; StAndré PB40695; between St-Benoit and SteRose KK33459. Corticolous and saxicolous.

Haematomma persoonii (Fée) A.Massal. - SteRose PB40151. Corticolous.

Hemithecium aphanes (Mont. \& Bosch) M.Nakan. \& Kashiw. - Grand Étang PB40078. Corticolous.

Herpothallon albidum (Fée) Aptroot, Lücking \& G.Thor - Between St-Denis and Plaine des Chicots DE4830. Corticolous. 
Herpothallon roseocinctum (Fr.) Aptroot, Lücking \& G.Thor - Between St-Denis and Plaine des Chicots DE4813. Corticolous.

Heterodea madagascareum Nyl. - Forêt de Bébour PB40530, LG; Cirque de Cilaos MB59773. Terricolous and corticolous. This conspicuous species is not close to other species of the genus Heterodea and its real affinity is currently under study by E. Timdal.

Heterodermia appendiculata (Kurok.) Swinscow \& Krog - Between St-Benoit and Ste-Rose KK26147. Corticolous.

Heterodermia comosa (Eschw.) Follmann \& Redon - Cirque de Salazie PB40936. Corticolous and over old leathery leaves.

Heterodermia dissecta (Kurok.) D.D.Awasthi Cirque de Cilaos KK26444. Corticolous.

Heterodermia flabellata (Fée) D.D.Awasthi Piton de la Fournaise GF (B 60 0128923). Corticolous.

Heterodermia fragilissima (Kurok.) J.C.Wei \& Y.M.Jiang - Cirque de Cilaos KK33257; Plaine d'Affouches KK33752; between St-Benoit and Ste-Rose KK33639. Corticolous.

Heterodermia hypoleuca (Mühl.) Trevis. - Le Tampon PB40818; Plaine des Palmistes PB40298. Corticolous.

Heterodermia isidiophora (Vain.) D.D.Awasthi Cirque de Cilaos KK26280. Corticolous.

Heterodermia japonica (Sato) Swinscow \& Krog - Cirque de Cilaos US1597, KK26353; Forêt de Bébour PB39814, LG; Piton la Fournaise PB40657, KK33709. Corticolous.

Heterodermia koyana (Kurok.) Elix - Cirque de Cilaos KK33368, 33369. Corticolous. An interesting addition to the lichen flora of Africa as this species was formerly known from Central America, Asia and Australia (Elix 2010).

Heterodermia leucomelos (L.) Poelt - Cirque de Cilaos US1425; Cirque de Salazie PB40941; Forêt de Bébour PB39924; Grand Étang DM974.1494; Piton de la Fournaise GF (B 60 0128913); Plaine des Palmistes PB40301; StAndré DM974.1816; Ste-Marie DM974.0444. Corticolous.

Heterodermia leucomelos subsp. boryi (Fée) Swinscow \& Krog - Cirque de Cilaos US1543, KK33678; Forêt de Bébour PB40332; Piton de la Fournaise GF (B 60 0135149); Plaine des Palmistes PB39867; between St-Benoit and SteRose KK33638. Corticolous.
Heterodermia lutescens (Kurok.) Follmann Bras-Panon DM974.1738; Cirque de Cilaos KK33673; Cirque de Salazie DM974.0327; Forêt de Bébour DM974.0028; St-André DM974.1808. Corticolous and saxicolous.

Heterodermia microphylla (Kurok.) Skorepa - Cirque de Cilaos LG, KK26448; Plaine d'Affouches KK26396. Corticolous.

Heterodermia obscurata (Nyl.) Trevis. - Cirque de Cilaos KK33682; Forêt de Bébour PB40333; between St-Denis and la Possession KK33758. Corticolous.

Heterodermia podocarpa (Bél.) D.D.Awasthi Forêt de Bébour PB39803; Plaine des Palmistes PB40294. Corticolous.

Heterodermia pseudospeciosa (Kurok.) W.L.Culb. - Cirque de Cilaos PB40200, KK26150; Forêt de Bébour PB39765; St-André PB40694. Saxicolous.

Heterodermia reagens (Kurok.) Elix - Cirque de Cilaos KK25484; Cirque de Salazie KK26568; Piton de la Fournaise KK26316. Corticolous.

Heterodermia speciosa (Wulfen) Trevis. - Forêt de Bébour KK33618. Saxicolous.

Heterodermia vulgaris (Vain.) Follmann \& Redón - St-Paul DM974.0263. Muscicolous on rock.

Hyperphyscia adglutinata (Flörke) H.Mayrhofer \& Poelt - St-Benoit KK33614; St-Joseph PB40595. Corticolous.

Hyperphyscia pandani (H.Magn.) Moberg Cirque de Salazie PB40734. Corticolous.

Hypotrachyna afrorevoluta (Krog \& Swinscow) Krog \& Swinscow - Le Tampon DM974.1581. Corticolous.

Hypotrachyna endochlora (Leight.) Hale Cirque de Cilaos US1598; Cirque de Mafate DM974.1789; Cirque de Salazie DM974.0306, KK25465; Forêt de Bébour DM974.0401; Plaine des Palmistes DM974.1465; St-Paul DM974.1918. Corticolous and muscicolous.

Hypotrachyna evansii Knox - Cirque de Cilaos KK26019; Cirque de Mafate DM974.1704; Cirque de Salazie DM974.0307; Forêt de Bébour DM974.0371; Le Tampon DM974.1400. Corticolous. A species originally described from Transvaal in South Africa (KNox 1982). Hypotrachyna olivetorica Krog described from Reunion (KroG 2000) and also reported from Tanzania is most probably a synonym: all details in the description (including the non-sorediate pustules and production of olivetoric acid) match between both species. 
Hypotrachyna imbricatula (Zahlbr.) Hale - Cirque de Salazie DM974.0340; Forêt de Bébour DM974.1663; Plaine des Palmistes PB39757; Ste-Marie DM974.0454. Corticolous.

Hypotrachyna immaculata (Kurok.) Hale - Cirque de Mafate DM974.1760; Piton de la Fournaise PB39990; between St-Benoit and Ste-Rose KK26142. Corticolous.

Hypotrachyna laevigata (Sm.) Hale - Cirque de Cilaos KK25945. Corticolous.

Hypotrachyna leiophylla (Kurok.) Hale - Le Tampon PB40746; Plaine des Palmistes PB39881. Corticolous.

Hypotrachyna microblasta (Vain.) Hale Cirque de Cilaos KK26443; Cirque de Salazie DM974.0308; Forêt de Bébour DM974.0413; St-Paul DM974.1919; St-Philippe GF (B 60 0135425). Corticolous.

Hypotrachyna orientalis (Hale) Hale - Cirque de Cilaos US1532, KK26296; Cirque de Mafate DM974.1795; Cirque de Salazie KK33699; Forêt de Bébour DM974.1536; Forêt du Tévelave DM974.0283; Le Tampon DM974.1402; St-Paul DM974.0274; Ste-Rose DM974.1593. Corticolous.

Hypotrachyna osseoalba (Vain.) Park \& Hale Cirque de Cilaos KK26431; Cirque de Mafate DM974.1759; Forêt de Bébour PB40450; Forêt de Bélouve KK25487; Le Tampon DM974.1619; Piton de la Fournaise KK26312; Plaine des Palmistes PB39758; St-Paul DM.9740259; SteRose DM974.1599. Corticolous and saxicolous. Hypotrachyna polydactyla (Krog) Nash - Cirque de Cilaos KK26293. Corticolous.

Hypotrachyna pseudosinuosa (Asah.) Hale Cirque de Mafate DM.974.1791; Forêt de Bébour DM974.1535; St-André DM974.1807; St-Paul DM974.0277; Ste-Rose DM974.1617. Corticolous.

Hypotrachyna revoluta (Flörke) Hale - Piton Doré to Piton Textor KK3585. Corticolous.

Hypotrachyna rockii (Zahlbr.) Hale - Cirque de Cilaos PB40194, KK33749; Forêt de Bébour DM974.0115; Plaine des Palmistes PB40905. Corticolous and saxicolous.

Hypotrachyna sinuosa (Sm.) Hale - Forêt de Bébour PB40495; Le Tampon DM974.1577; Piton de la Fournaise PB39957; St-Paul DM974.0229. Corticolous.

Imshaugia aleurites (Ach.) S.L.F.Mey. - Forêt de Bébour PB40510, LG. Corticolous.
Kroswia crystallifera P.M.Jørg. - Cirque de Cilaos KK33707; Forêt de Bébour PB40435, LG. Corticolous.

Lecanora caesiorubella Ach. - Cirque de Cilaos US1463; Forêt de Bébour LG. Corticolous.

Lecanora flavopallida Stirt. - Grand Étang KK25518; St-André PB40698. Corticolous.

Lecanora jamesii J.R.Laundon - Piton de la Fournaise KK26323. Corticolous.

Lecanora oreinoides (Körb.) Hertel \& Rambold St-Gilles-les-Bains KK26555. Saxicolous.

Lecanora subravida Nyl. - Piton de la Fournaise PB40020. Corticolous.

Lecidella punctuliformis (Nyl.) Kalb - Piton Maïdo KK26414; between St-Benoit and SteRose KK26133. Corticolous.

Leioderma erythrocarpum (Delise ex Nyl.) D.J.Galloway \& P.M.Jørg. - Cirque de Cilaos US1652; Cirque de Salazie KK25482; Forêt de Bébour PB39925, LG; Grand Étang PB40024, KK25515; Piton Doré to Piton Textor KK25512; Plaine des Palmistes PB39871; StAndré PB40726; Ste-Marie DM974.0458. Corticolous.

Leioderma sorediatum D.J.Galloway \& P.M.Jørg. - Forêt de Bébour PB40556, LG; Plaine des Palmistes PB40864. Corticolous.

Leiorreuma exaltatum (Mont. \& Bosch) Staiger Forêt de Bébour PB40513. Corticolous.

Leiorreuma hypomelaenum (Müll.Arg.) Staiger Forêt de Bébour PB40314; Plaine des Palmistes PB40875. Corticolous.

Leiorreuma sericeum (Eschw.) Staiger - Ste-Rose PB40137. Corticolous.

Lepraria jackii Tønsberg - Cirque de Cilaos KK33666. Corticolous.

Lepraria sipmaniana (Kümmerl. \& Leuckert) Kukwa - Cirque de Cilaos US1429. Corticolous. Lepraria yunnaniana (Hue) Zahlbr. - Forêt de Bébour PB40321. Plaine des Palmistes PB39888. Corticolous and terricolous.

Leprocaulon arbuscula (Nyl.) Nyl. - Forêt de Bébour PB40359; Plaine des Palmistes PB40913; between St-Benoit and Ste-Rose KK33783; between St-Denis and Plaine des Chicots DE4769. Corticolous and muscicolous.

Leptogium austroamericanum (Malme) C.W. Dodge - Cirque de Salazie DM974.0348; Grand Étang DM974.1493; St-André DM974.1819; St-Philippe DM974.0215. Corticolous. 
Leptogium azureum (Ach.) Mont. - Cirque de Cilaos US1504, KK33655; Forêt de Bébour KK33683; Forêt Domaniale du Grand Brûlé KK33543; Grand Étang PB40023; Piton de la Fournaise GF (B 60 0135427); Plaine des Palmistes PB39726; St-Benoît PB40994; between St-Benoit and Ste-Rose KK33642; StPhilippe GF (B 60 0135451). Corticolous.

Leptogium burnetiae C.W.Dodge - Cirque de Cilaos KK33546. Corticolous.

Leptogium coralloideum (Meyen \& Flot.) Vain. - Cirque de Cilaos US1524, KK33723; Forêt de Bébour DM974.1637; Le Tampon DM974.1408; St-André PB40689; between StBenoit and Ste-Rose KK33364. Corticolous.

Leptogium cyanescens (Rabenh.) Körb. Cirque de Cilaos US1458; Cirque de Salazie DM974.0351; Forêt de Bébour DM974.0153; Piton de la Fournaise GF (B 60 0128926); St-Benoît PB40999; St-Philippe PB40575. Corticolous.

Leptogium hibernicum P.M.Jørg. - Cirque de Mafate DM.9741708; Cirque de Salazie DM974.0332; Forêt de Bébour DM974.1834. Corticolous.

Leptogium laceroides de Lesd. - Cirque de Cilaos US1491; Cirque de Mafate DM974.1700; Cirque de Salazie DM974.0333; Forêt de Bébour DM974.0366; Le Tampon DM974.1572; Piton de la Fournaise GF (B 60 0135103); Piton Doré to Piton Textor KK33573. Corticolous.

Leptogium marginellum (Sw.) Gray - Cirque de Salazie KK33652. Corticolous.

Leptogium phyllocarpum (Pers.) Mont. - Cirque de Cilaos US1476; Cirque de Salazie KK25503; Forêt de Bébour PB40378, LG. Corticolous.

Letrouitia vulpina (Tuck.) Hafellner \& Bellem. - Cirque de Cilaos KK26435; Grand Étang KK25516; Piton de la Fournaise GF (B 60 0128907); Plaine d'Affouches KK26008. Corticolous.

Leucodecton fissurinum (Hale) A.Frisch - Cirque de Cilaos KK25978; Plaine des Palmistes KK26556. Corticolous.

Lithogyalideopsis zeylandica (Vězda \& Malcolm) Lücking, Sérus. \& Vězda — Plaine des Palmistes PB40616. Saxicolous. An inconspicuous but typical species, formerly only known from New Zealand (VĚZDA \& MALCOLM 1997).

Lobaria asperula (Stirt.) Yoshim. - Cirque de Cilaos PB40289, US1494. Corticolous. A rare species, close to $L$. discolor and $L$. patinifera, formerly known from New Zealand (GALloway 2007; there considered as endemic to New Zealand) and Tanzania (Krog 2000).
Lobaria discolor (Delise) Hue - Cirque de Cilaos PB40285, LG; Cirque de Mafate DM974.1711; Cirque de Salazie DM974.0337; Forêt de Bébour LG; Plaine des Palmistes PB39742. Corticolous.

Lobaria isidiosa (Müll.Arg.) Vain. - Cirque de Cilaos US1513; Piton de la Fournaise GF (B 60 0128948). Corticolous.

Lobaria patinifera (Taylor) Hue - Cirque de Cilaos US1662, LG. Corticolous.

Lobaria pulmonaria (L.) Hoffm. - Cirque de Cilaos US1496, LG; Le Tampon DM974.1433; Plaine des Palmistes PB39898. Corticolous.

Lobaria retigera (Bory) Trevis. - Cirque de Cilaos US1567, LG; Cirque de Salazie DM974.0328; Forêt de Bébour PB40367, LG; Grand Étang PB40031; Piton de la Fournaise GF (B 60 0128949, B 60 0142003, B 60 0142004); Plaine des Palmistes DM974.1469; Ste-Marie DM974.0453. Corticolous.

Malmidea ceylanica (Zahlbr.) Kalb, Rivas Plata \& Lumbsch - Grand Étang PB40027. Corticolous. Malmidea granifera (Ach.) Kalb, Rivas Plata \& Lumbsch - Grand Étang PB40035; Plaine des Palmistes PB39738, LG. Corticolous.

Mazosia dispersa (Hedrick) R.Sant. - Cirque de Cilaos LG, DE4561. Foliicolous.

Mazosia phyllosema (Nyl.) Zahlbr. - Forêt de Bébour PB40334; between St-Denis and Plaine des Chicots DE4811. Bambusicolous.

Megalaria laureri (Hepp ex Th.Fr.) Hafellner - Cirque de Cilaos US1481; Forêt de Bébour PB40365; Le Tampon PB40764; Plaine des Palmistes PB39834. Corticolous.

Megalaria pulverea (Borrer) Hafellner \& E.Schreiner - Forêt de Bébour PB39930; Piton de la Fournaise PB39997. Corticolous.

Megaloblastenia marginiflexa (Hook.f. \& Taylor) Sipman var. dimota (Malme) Sipman - Cirque de Cilaos US1479; Cirque de Salazie KK33736; Forêt de Bébour PB40364; Plaine des Palmistes PB39832; between St-Benoit and Ste-Rose KK26167. Corticolous. This subspecies is much more common in the Neotropics than the few reports in SiPMAN (1983) suggest, and is here also reported as new for Africa and the palaeotropics.

Megalospora atrorubicans (Nyl.) Zahlbr. Cirque de Cilaos KK26380; Cirque de Salazie PB40966; Piton de la Fournaise GF (B 60 0128906). Corticolous.

Megalospora coccodes (Bél.) Sipman - Cirque de Cilaos KK26021. Corticolous. 
Megalospora pruinata (Müll.Arg.) Sipman Grand Étang PB40028. Corticolous.

Megalospora sulphurata Meyen - Cirque de Cilaos US1445, KK26434; Forêt de Bébour PB40349, DE4151. Corticolous.

Megalospora tuberculosa (Fée) Sipman - Cirque de Cilaos US1457, KK26439; Piton Doré to Piton Textor KK33583; Plaine des Palmistes PB39847; between St-Benoit and Ste-Rose KK26164. Corticolous.

Melanotrema meiospermum (Nyl.) A.Frisch Piton de la Fournaise GF (B 60 0135413). Corticolous.

Menegazzia capitata Sipman \& Bjerke - Forêt de Bébour MB, LG. Corticolous. A species formerly known from Malaysia/Sabah and Tanzania (BJERKE \& SipMAN 2007).

Menegazzia subsimilis (H.Magn.) R.Sant. Forêt de Bébour PB40404, LG; Le Tampon DM974.1410; Piton Doré to Piton Textor KK33622. Corticolous.

Musaespora kalbii Lücking \& Sérus. - St-André PB40719, LG. Foliicolous.

Mycomicrothelia confluens (Müll.Arg.) D.Hawksw. - Cirque de Cilaos US1478. Corticolous.

Myelochroa aurulenta (Tuck.) Elix \& Hale Cirque de Cilaos PB40204. Overgrowing saxicolous mosses.

Myelochroa denegans (Nyl.) Elix \& Hale - BrasPanon DM974.1748. Corticolous.

Myeloconis erumpens P.M.McCarthy \& Elix - Cirque de Cilaos KK26031. Corticolous. First mention of this genus for Africa, otherwise known from the Neotropics and tropical Australasia (McCARThy \& Elix 1996); also known from continental Africa (E. Sérusiaux, unpublished records).

Myriotrema hartii (Müll.Arg.) Hale - Between St-Benoit and Ste-Rose KK25052. Corticolous.

Myriotrema microporum (Mont.) Hale - Grand Étang PB40041; Piton de la Fournaise GF (B 60 0128919). Corticolous.

Nephroma tropicum (Müll.Arg.) Zahlbr. - Cirque de Cilaos US1566, KK33672; Forêt de Bébour, LG; Plaine des Palmistes PB40880. Corticolous.

Normandina pulchella (Borrer) Nyl. - Forêt de Bébour PB40500; between St-Denis and Plaine des Chicots DE4826. Corticolous.

Ocellularia postposita (Nyl.) A.Frisch - Forêt de Bébour KK25198; Piton Doré to Piton Textor KK33596; Plaine des Palmistes PB39759; between St-Benoit and Ste-Rose KK33360. Corticolous.
Ocellularia subsimilis (Hale) Hale - Grand Étang KK25525. Corticolous.

Ochrolechia africana Vain. - Cirque de Cilaos KK33668. Corticolous.

Ochrolechia androgyna (Hoffm.) Arnold-Between St-Benoit and Ste-Rose KK26390. Corticolous.

Opegrapha trilocularis Müll.Arg. - Cirque de Cilaos US1545. Corticolous.

Pannaria elatior Stirt. - Plaine d'Affouches LG. Corticolous.

Pannaria lurida (Mont.) Nyl. - Cirque de Cilaos MB59790; Forêt de Bébour PB40439, LG. Corticolous.

Pannaria multifida P.M.Jørg. - Cirque de Cilaos KK33373; Forêt de Bébour PB40362, LG; Grand Étang PB40033, LG, KK25513; between St-Benoit and Ste-Rose KK26388. Corticolous.

Pannaria rubiginosa (Ach.) Bory - Cirque de Cilaos US1499, KK33708; Cirque de Salazie KK25488; Forêt de Bébour PB2340439, LG; Piton de la Fournaise GF (B 60 0128945); Piton Doré to Piton Textor KK33598; between StBenoit and Ste-Rose KK33438. Corticolous.

Pannaria sphinctrina (Mont.) Hue - Grand Étang KK26504. Corticolous.

Pannaria squamulosa P.M.Jørg. - Cirque de Cilaos MB59911; Forêt de Bébour PB40330; Plaine des Palmistes PB39873, MB58269. Corticolous and overgrowing saxicolous mosses. A species formerly known only from South Africa (JøRGENSEN 2003).

Paraparmelia annexa (Kurok.) Elix \& J.Johnston - Piton de la Fournaise GF (B 60 0128966). Saxicolous.

Parmeliella brisbanensis (C.Knight) P.M.Jørg. \& D.J.Galloway - Piton de la Fournaise GF (B 60 0128920, B 60 0135439); between St-Benoit and Ste-Rose KK25992; St-Philippe LG, GF (B 60 0135428). Corticolous.

Parmeliella cinerata (Zahlbr.) P.M.Jørg. La Roche Ecrite LG; St-Benoît PB41002. Corticolous.

Parmeliella mariana (Fr.) P.M.Jørg. - St-Philippe LG; Ste-Rose MB58545. Corticolous.

Parmeliella pannosa (Sw.) Müll.Arg. - Cirque de Cilaos US1423; Piton de la Fournaise GF (B 60 0135105). No 1423 teste P.M. Jørgensen.

Parmeliella polyphyllina P.M.Jørg. - Cirque de Cilaos LG; Forêt de Bébour PB40381; Piton de la Fournaise PB39998; St-André PB40709; St-Philippe LG. Corticolous. A species formerly known from Australia/Queensland, Papua New Guinea and Indonesia/Java (JøRGENSEN 2001). 
Parmeliella stylophora (Vain.) P.M.Jørg. - StBenoît PB41001; St-Philippe PB40558, LG. Corticolous.

Parmelinopsis damaziana (Zahlbr.) Elix \& Hale - Cirque de Cilaos KK33706; Le Tampon PB40759; Piton de la Fournaise PB40004; Piton Maïdo KK25949; St-Paul DM974.0266. Corticolous.

Parmelinopsis minarum (Vain.) Elix \& Hale Cirque de Cilaos US1532; Forêt de Bébour PB40429; Plaine des Palmistes PB39794. Corticolous

Parmelinopsis subfatiscens (Kurok.) Elix \& Hale - Forêt de Bébour PB39940; Le Tampon DM974.1579; Ste-Rose DM974.1607. Saxicolous and corticolous.

Parmotrema austrosinense (Zahlbr.) Hale - Le Tampon PB40807. Corticolous.

Parmotrema cooperi (J.Steiner \& Zahlbr.) Sérus. - St-Paul DM974.1911. Corticolous.

Parmotrema crinitum (Ach.) M.Choisy Cirque de Cilaos PB40214; Cirque de Salazie DM974.0297; Forêt de Bébour DM974.0023; Forêt du Tévelave DM974.0284; Le Tampon PB40776; Piton de la Fournaise PB40679; Plaine des Palmistes PB39744; St-Paul DM974.1923; Ste-Rose DM974.1618. Corticolous.

Parmotrema cristiferum (Taylor) Hale - Cirque de Salazie DM974.0432; Ste-Rose PB40147. Corticolous and saxicolous.

Parmotrema gardneri (C.W.Dodge) Sérus. - Piton de la Fournaise GF (B 60 0135114); Plaine des Palmistes PB39783. Corticolous.

Parmotrema mellissii (C.W.Dodge) Hale - Cirque de Mafate DM974.1768. Corticolous.

Parmotrema paulense (Zahlbr.) Hale - Forêt de Bébour PB40489. Corticolous.

Parmotrema praesorediosum (Nyl.) Hale - Piton de la Fournaise GF (B 60 0128928); St-Benoît PB40992. Corticolous.

Parmotrema reticulatum (Taylor) M.Choisy Cirque de Cilaos US1440, DE4639; Cirque de Mafate DM974.1786; Cirque de Salazie DM974.0295; Forêt de Bébour PB40897; Le Tampon PB40809; Piton de la Fournaise GF (B 60 0128930); Plaine des Palmistes PB39915; StBenoît PB40993; Ste-Marie DM974.0447; StPaul DM974.0234; St-Philippe DM974.0212. Corticolous and saxicolous.

Parmotrema robustum (Degel.) Hale - Forêt de Bébour PB40520. Corticolous.
Parmotrema subisidiosum (Müll.Arg.) Hale Cirque de Cilaos US1495; Plaine des Palmistes PB39763. Corticolous and saxicolous.

Parmotrema tinctorum (Nyl.) Hale - Cirque de Cilaos US1442; Cirque de Mafate DM974.1794; Cirque de Salazie PB40942; Forêt de Bébour DM974.0415; Forêt du Tévelave DM974.0287; Le Tampon PB40804; Plaine des Palmistes PB39864; St-André DM974.1804; St-Paul DM974.1863; St-Philippe GF (B 60 0135117); Ste-Rose DM974.0290. Corticolous and saxicolous.

Parmotrema zollingeri (Hepp) Hale - Cirque de Cilaos US1453; St-Philippe PB40567. Corticolous.

Peltigera dolichorhiza (Nyl.) Nyl. - Cirque de Cilaos US1507, LG, KK33663; Cirque de Salazie KK33717; Forêt de Bébour PB40316, LG; Plaine des Palmistes PB39904; between StBenoit and Ste-Rose KK33646. Corticolous and over saxicolous mosses.

Peltigera neckeri Hepp ex Müll.Arg. - Cirque de Cilaos US1428, LG. Terricolous.

Peltigera polydactyloides Nyl. - Cirque de Cilaos US1497, LG, KK33553; between St-Benoit and Ste-Rose KK33372. Terricolous.

Peltigera polydactylon (Neck.) Hoffm. - Cirque de Cilaos KK33731; between St-Benoit and Ste-Rose KK33379. Muscicolous.

Peltigera praetextata (Sommerf.) Zopf - Cirque de Cilaos US1500 LG, KK33726; Piton de la Fournaise GF (B 60 0128940). Terricolous.

Peltigera sorediifera (Nyl.) Vitik. - Plaine d'Affouches LG. Terricolous.

Peltigera ulcerata Müll.Arg. - Cirque de Cilaos LG, KK33664; Forêt de Bébour LG. Terricolous.

Peltula obscurans (Nyl.) Gyeln. - Cirque de Cilaos PB40184; Le Tampon PB40853. StGilles-les-Bains KK26518. Saxicolous.

Pertusaria aspera Müll.Arg. - Cirque de Cilaos KK33767. Corticolous.

Pertusaria subventosa Malme var. hypothamnolica Archer \& Elix - Cirque de Cilaos KK25551. Saxicolous.

Phaeographis atromaculata Archer - Le Tampon PB40813. Corticolous.

Phaeographis brasiliensis (A.Massal.) Kalb \& Matthes-Leicht - Ste-Rose PB40103. Corticolous.

Phaeographis caesiodisca Staiger - Forêt de Bébour PB40426. Corticolous. 
Phaeographis dendritica (Ach.) Müll.Arg. - Cirque de Salazie PB40937; Le Tampon PB40828; Plaine des Palmistes PB40306. Corticolous.

Phaeographis intricans (Nyl.) Staiger - Plaine des Palmistes PB39754. Corticolous.

Phaeographis leiogrammodes (Kremp.) Müll.Arg. - Plaine des Palmistes PB40302. Corticolous.

Phaeographis lobata (Eschw.) Müll.Arg. - Forêt de Bébour LG. Corticolous.

Phaeographis nylanderi (Vain.) Zahlbr. - Plaine des Palmistes PB39857. Corticolous.

Phaeographis scalpturata (Ach.) Staiger - Cirque de Cilaos PB40254. Corticolous.

Phlyctidia boliviensis (Nyl.) Müll.Arg. - Plaine des Palmistes PB39850. Corticolous.

Phyllopsora albicans Müll.Arg. - St-Benoît PB41008. Corticolous.

Phyllopsora borbonica Timdal \& Krog Grand Étang PB40040, LG; Plaine des Palmistes PB40909; St-André PB40702; StBenoît PB41010; St-Philippe PB40579, LG. Corticolous.

Phyllopsora buettneri (Müll.Arg.) Zahlbr. Cirque de Cilaos PB40235, LG; Grand Étang PB40060. Corticolous.

Phyllopsora chlorophaea (Müll.Arg.) Zahlbr. Cirque de Cilaos PB40256, LG; Le Tampon LG; Piton de la Fournaise LG; Plaine d'Affouches LG. Corticolous.

Phyllopsora confusa Swinscow \& Krog - Piton de la Fournaise PB39984; Plaine des Palmistes PB40067, LG; St-Joseph LG. Corticolous.

Phyllopsora furfuracea (Pers.) Zahlbr. - Cirque de Cilaos LG; Grand Étang PB40059; St-André PB40708. Corticolous.

Phyllopsora nemoralis Timdal \& Krog - StPhilippe PB40587. Corticolous.

Phyllopsora subhispidula (Nyl.) Kalb \& Elix Cirque de Cilaos PB40257. Corticolous.

Phyllopsora swinscowii Timdal \& Krog - StAndré PB40723. Corticolous.

Physcia alboplumbea (Taylor) Nyl. - Forêt de Bébour KK33615. Saxicolous

Physcia atrostriata Moberg - St-Philippe PB40169; Ste-Rose PB40126. Corticolous.

Physcia krogiae Moberg - St-Paul DM974.0231. Corticolous.

Physcia poncinsii Hue - St-Benoit KK33612. Corticolous.

Physcia undulata Moberg - Cirque de Salazie KK26463; St-Benoit KK33611. Saxicolous.
Physcidia wrightii (Tuck.) Tuck. - Cirque de Salazie DM974.0310; Grand Étang PB40032, LG; St-Benoît PB41006; St-Paul DM974.1856. Corticolous.

Physma byrsaeum (Ach.) Tuck. - Grand Étang PB40029, LG; Piton de la Fournaise GF (B 60 0128931), US1714; St-Benoît PB41000; StPhilippe PB40570, GF (B 60 0135456), LG. Corticolous.

Piccolia elmeri (Vain.) Hafellner - Cirque de Cilaos PB40240; Le Brûlé KK38915; Le Tampon PB40751; between St-Benoit and SteRose KK33597. Corticolous.

Placopsis parellina (Nyl.) I.M.Lamb - Piton Maïdo KK25948; Plaine d'Affouches KK25509. Saxicolous.

Placynthiella dasaea (Stirt.) Tønsberg - Le Tampon PB40841. Corticolous.

Platythecium leiogramma (Nyl.) Staiger - Cirque de Cilaos KK25971. Corticolous.

Polychidium dendriscum (Nyl.) Henssen - Piton de la Fournaise GF (B 60 0133246). Corticolous.

Polymeridium catapastum (Nyl.) R.C.Harris Cirque de Cilaos US1631. Corticolous.

Porina aenea (Wallr.) Zahlbr. - Bois de Nèfles MB60066. Corticolous.

Porina albicera (Kremp.) Overeem - St-Benoît PB41025. Foliicolous.

Porina atrocoerulea Müll.Arg. - Cirque de Cilaos LG. Foliicolous.

Porina decrescens P.M.McCarthy \& Kantvilas - Piton de la Fournaise PB39969, MB58354. Corticolous.

Porina epiphylla (Fée) Fée - Cirque de Salazie PB40961; Forêt de Bébour PB39906, 39907; St-Benoît MB41024. Foliicolous.

Porina farinosa Knight - Cirque de Cilaos PB40244, MB58640. Corticolous.

Porina internigrans (Nyl.) Müll.Arg - Bois de Nèfles MB60009; Le Tampon MB59166; Plaine des Palmistes PB39728. Corticolous.

Porina leptalea (Durieu \& Mont.) A.L.Sm. Le Tampon PB40802; Piton de la Fournaise MB58380. Corticolous.

Porina lucida R.Sant. - Grand Étang PB40049; Plaine des Palmistes PB40911. Foliicolous.

Porina nitidula Müll.Arg. - Cirque de Salazie PB40923. Foliicolous.

Porina nucula Ach. - Grand Étang PB40081; Plaine des Palmistes PB39743; St-André PB40705. Corticolous.

Porina mastoidea (Ach.) Müll.Arg. - St. Joseph PB40610. Saxicolous. 
Porina riparia P.M.McCarthy - Bois de Nèfles MB59437; Cirque de Cilaos MB 59611; Plaine des Palmistes MB59012. Saxicolous.

Porina rufula (Kremp.) Vain. - Cirque de Cilaos LG. Foliicolous

Porina sagedioides (Zahlbr.) P.M.McCarthy Bois de Nèfles MB59416. Saxicolous.

Porina tetracerae (Afz. in Ach.) Müll.Arg. Cirque de Cilaos PB40206; St-Joseph PB40603. Saxicolous.

Pseudocyphellaria argyracea (Delise) Vain. Cirque de Cilaos US1713, LG; Cirque de Salazie DM974.0316; Forêt de Bébour PB40337, LG; Grand Étang PB40074; Piton de la Fournaise GF (B 60 0128927); St-Philippe DM974.0211; Ste-Rose PB40043. Corticolous.

Pseudocyphellaria aurata (Ach.) Vain. - Cirque de Cilaos US1527, LG; Cirque de Mafate DM974.0468; Cirque de Salazie DM974.0298; Forêt de Bébour PB39935, LG; Piton de la Fournaise GF (B 60 0128912); St-André DM974.1820; St-Benoît PB41014; St-Philippe GF (B 60 0135108); Ste-Marie DM974.0466. Corticolous.

Pseudocyphellaria clathrata (De Not.) Malme Cirque de Cilaos LG. Corticolous.

Pseudocyphellaria crocata (L.) Vain. - BrasPanon DM974.1736; Cirque de Cilaos PB40210, US1447, LG; Cirque de Mafate DM974.1699; Cirque de Salazie DM974.0299; Forêt de Bébour PB40437, LG; Grand Étang PB40044; Le Tampon DM974.1445; Piton de la Fournaise GF (B 60 0128909); Plaine des Palmistes PB40855; St-André DM974.1824; St-Philippe GF (B 60 0128973). Corticolous and over saxicolous mosses.

Pseudocyphellaria desfontainii (Delise) D.J.Galloway - Forêt de Bébour PB40366, LG; Grand Étang DM974.1502; Le Tampon DM974.1443; Plaine des Palmistes PB39858; St-André DM974.1817. Corticolous.

Pseudocyphellaria dozyana (Mont. \& Bosch) D.J.Galloway - Cirque de Cilaos LG; Forêt de Bébour, LG; Grand Étang DM974.1501; Plaine d'Affouches DM974.1952; St-André DM974.1827. Corticolous.

Pseudoparmelia sphaerospora (Nyl.) Hale Cirque de Mafate DM974.1764. Corticolous.

Psoroglaena stigonemoides (Orange) Henssen Piton de la Fournaise LG. Corticolous.

Psoroma subhispidulum Nyl. - Forêt de Bébour, LG. Corticolous.
Pyrenula dermatodes (Borrer) Schaer. - Forêt de Bébour PB39809; St-Philippe PB40172. Corticolous.

Pyrenula duplicans (Nyl.) Aptroot - Cirque de Cilaos KK26030. Corticolous.

Pyrenula massariospora (Starbäck) R.C.Harris Cirque de Cilaos PB40271. Corticolous.

Pyrenula microcarpa Müll.Arg. - Cirque de Cilaos US1705. Corticolous.

Pyrenula nitidula (Bres.) R.C.Harris - Plaine des Palmistes PB39727; St-André PB40696; StPhilippe PB40172. Corticolous.

Pyrenula ochraceoflava (Nyl.) R.C.Harris - Piton de la Fournaise GF (B 60 0128944); St-Philippe PB41051. Corticolous.

Pyrenula parvinuclea (Meyen \& Flot.) Aptroot St-Philippe PB40167. Corticolous.

Pyrenula quassiaecola Fée - Cirque de Cilaos PB40277; Plaine des Palmistes PB39916; StPhilippe PB40589. Corticolous.

Pyxine cognata Stirt. - Cirque de Cilaos KK25550. Saxicolous.

Pyxine coralligera Malme - Ste-Rose PB40111. Corticolous and saxicolous.

Pyxine sorediata (Ach.) Mont. - Cirque de Cilaos PB40262, US1493, KK26281; Cirque de Mafate DM974.1713; Piton de la Fournaise GF (B 60 0135106). Corticolous and saxicolous.

Pyxine subcinerea Stirt. - Between St-Benoit and Ste-Rose KK33613; St-Joseph PB40598. Corticolous.

Rhabdodiscus fissus (Nyl.) Vain. - Cirque de Cilaos KK33806. Corticolous.

Rhizocarpon geographicum (L.) DC. - Piton de la Fournaise GF (B 60 0128962). Saxicolous.

Rinodina connectens Malme - Cirque de Cilaos MB59638; Cirque de Salazie MB59223; Piton de la Fournaise MB59064. Corticolous.

Rinodina dolichospora Malme - La Grande Chaloupe MB59358. Corticolous.

Rinodina isidioides (Borrer) H.Olivier - Piton de la Fournaise MB58396, PB40642. Corticolous.

Rinodina oxydata (A.Massal.) A.Massal. - Cirque de Cilaos MB59592, US1431; Étang-Salé MB59952; La Grande Chaloupe MB59351; Le Tampon PB40852; Plaine des Palmistes MB59254; St-Joseph MB59000; Ste-Rose MB58502. Saxicolous.

Rinodina xanthomelana Müll.Arg. - Cirque de Cilaos MB59601, KK26344. Saxicolous.

Sclerophyton elegans Eschw. - Cirque de Cilaos US1539. Corticolous. 
Siphula mascarena Mathey - Cirque de Cilaos US1610; Forêt de Bébour PB40094, LG; Forêt de Bélouve KK33694; Grand Étang PB40440; Le Brûlé KK33729; Piton Doré to Piton Textor KK33707; between St-Benoit and Ste-Rose KK26012. Corticolous and saxicolous.

Solenopsora elixiana Verdon \& Rambold - Forêt de Bébour MB58299. Saxicolous.

Solorina simensis Hochst. ex Flot. - Cirque de Cilaos DE4501, US1729, LG. Terricolous.

Sporopodiopsis mortimeriana Sérus. - Forêt de Bébour LG. Overgrowing corticolous mosses. Species previously only known from the type locality in Papua New-Guinea (SÉrusiaux 1997).

Spilonema revertens Nyl. - Cirque de Cilaos MB 59657. Saxicolous.

Spilonema schmidtii (Vain.) Henssen - Forêt de Bébour MB58943. Saxicolous.

Stereocaulon meyeri B.Stein - Cirque de Cilaos US1572; Piton de la Fournaise PB40684. Saxicolous.

Stereocaulon ramulosum Räuschel - Cirque de Cilaos US1503. Saxicolous.

Stereocaulon salazinum (Bory) Fée - Piton de la Fournaise GF (B 60 0135096). Saxicolous.

Stereocaulon scutelligerum Th.Fr. - Forêt de Bébour PB40391, LG; Petite-Ile LG; Piton de la Fournaise PB40687, GF (B 60 0128932), LG; St-Benoît PB41016; St-Philippe GF (B 60 0128934). Saxicolous.

Stereocaulon vesuvianum Pers. - Piton de la Fournaise GF (B 60 0135160, B 60 0128971); Plaine des Palmistes PB39876. Saxicolous.

Stereocaulon vulcani (Bory) Ach. - Piton de la Fournaise PB40681, LG. Saxicolous.

Sticta ambavillaria (Bory) Ach. - Cirque de Cilaos US1563, LG; Forêt de Bébour PB40329, LG; Plaine des Palmistes PB40895. Corticolous.

Sticta caperata (Nyl.) Nyl. - Cirque de Cilaos US1487, LG; Cirque de Salazie DM974.0303; Forêt de Bébour DM974.0043; Le Tampon PB40790; Piton de la Fournaise GF (B 60 0135112); Plaine des Palmistes PB39739; StAndré DM974.1815. Corticolous.

Sticta cyphellulata (Müll.Arg.) Hue - Cirque de Cilaos US1606, LG; Forêt de Bébour, LG; Grand Étang PB40026. Corticolous.

Sticta dichotoma Delise - Cirque de Cilaos US1505, LG; Cirque de Salazie DM974.302; Le Tampon DM974.1380; Piton des Neiges GF (B 60 0135113); Plaine des Palmistes PB39745; St-André DM974.1823; St-Benoît GF (B 60 0135118); St-Philippe GF (B 60 0135110). Corticolous.
Sticta fuliginosa (Hoffm.) Ach. - Cirque de Mafate DM974.1693; Cirque de Salazie DM974.0352; Forêt de Bébour PB39923, LG; Le Tampon DM974.1416; Plaine des Palmistes PB40878; St-Philippe GF (B 60 0135431). Corticolous.

Sticta macrophylla Delise - Bras-Panon DM974.1734; Forêt de Bébour DE4091; Grand Étang PB40030, LG; St-André DM974.1805. Corticolous and muscicolous.

Sticta orbicularis (A.Braun) Hue - Cirque de Cilaos US1489; Cirque de Mafate DM974.0472; Cirque de Salazie DM974.0318; Forêt de Bébour DM974.0056; Plaine des Palmistes DM974.1482; Le Tampon DM974.1417; StPhilippe DM974.0183. Corticolous.

Sticta plumbea Delise - Forêt de Bébour PB40361, LG; St-Philippe DM974.0208. Corticolous.

Sticta sublimbata (J.Steiner) Swinscow \& Krog - Cirque de Cilaos US1522; Cirque de Mafate DM974.1701; Cirque de Salazie DM974.0336; Forêt de Bébour LG; Grand Étang DM974.1506. Corticolous.

Sticta tomentosa (Sw.) Ach. - Cirque de Cilaos US1602, 1742; Cirque de Salazie DM974.0334; Forêt de Bébour DM974.0095; Grand Étang PB40075; Piton de la Fournaise GF (B 60 0135455); Plaine des Palmistes PB41047; StBenoît GF (B 60 0135116). Corticolous.

Sticta variabilis Ach. (= S. papyracea Delise) Cirque de Cilaos US1444, LG; Cirque de Mafate DM974.1709; Cirque de Salazie DM974.0329; Forêt de Bébour LG; Grand Étang PB40079, LG; Le Tampon DM974.1450; Piton de la Fournaise GF (B 60 0128941); Plaine des Palmistes PB39732, LG. Corticolous.

Sticta weigelii (Ach.) Vain. - Cirque de Cilaos US1523; Cirque de Mafate DM974.1705; Forêt de Bébour DM974.0161; Grand Étang DM974.1512; Le Tampon DM974.1419; Piton de la Fournaise GF (B 60 0128929); Plaine des Palmistes PB40867; St-André DM974.1829; St-Philippe GF (B 60 0128975). Corticolous.

Stigomatochroma metaleptoides (Nyl.) Marbach Forêt de Bébour LG. Corticolous.

Strigula nitidula Mont. - Cirque de Cilaos US1475, LG. Foliicolous.

Strigula phaea (Ach.) R.C.Harris - Plaine des Palmistes PB39879. Corticolous.

Strigula phyllogena (Müll.Arg.) R.C.Harris Cirque de Cilaos KK26370; Forêt de Bébour PB40369. Foliicolous.

Tapellaria phyllophila (Stirt.) R.Sant. - Cirque de Cilaos LG. Foliicolous. 
Teloschistes flavicans (Sw.) Norman - Cirque de Mafate DM974.1683; St-Paul DM974.1885. Corticolous.

Tephromela atra (Huds.) Hafellner - Ste-Rose PB40140. Corticolous.

Tephromela cerasina (Müll.Arg.) Rambold \& Triebel - Cirque de Cilaos PB40236. Overgrowing corticolous thallus of Dirinaria.

Thalloloma anguinum (Mont.) Trevis. - Grand Étang PB40070. Corticolous.

Thelotrema diplotrema Nyl. - Cirque de Cilaos KK33744; Cirque de Salazie KK33773; Plaine des Palmistes PB40615. Corticolous.

Thelotrema lepadinum (Ach.) Ach. - Cirque de Cilaos DE4637, PB40238, US1456; Cirque de Salazie KK33774; Forêt de Bébour PB39949; Piton de la Fournaise KK33693; Plaine des Palmistes PB39842. Corticolous and overgrowing saxicolous mosses.

Thelotrema porinoides Mont. \& Bosch - Grand Étang KK25511. Corticolous.

Thelotrema pachysporum Nyl. - Grand Étang PB40096. Corticolous.

Thelotrema subexpallescens Nagarkar, Sethy \& Patw. - Cirque de Salazie PB40988. Corticolous.

Toninia australis Timdal - Cirque de Salazie PB40950; St-Philippe DE4206; Ste-Rose PB40124. Saxicolous.

Trapelia coarctata (Sm.) M.Choisy - Forêt de Bébour PB40456; Grand Étang PB40077; Le Tampon PB40850. Saxicolous.
Trapeliopsis granulosa (Hoffm.) Lumbsch Cirque de Cilaos US1697; Forêt de Bébour PB40354. Corticolous and terricolous.

Tylophoron hibernicum (D.Hawksw., Coppins \& P.James) Ertz, Diederich, Bungartz \& Tibell (= Blarneya hibernica) - Cirque de Cilaos LG. Corticolous.

Usnea baileyi (Stirt.) Zahlbr. - Cirque de Cilaos US1454; Piton de la Fournaise GF (B 60 0135111); St-Philippe GF (B 60 0135415). Corticolous.

Usnea complanata (Müll.Arg.) Mot. - Cirque de Cilaos US1525. Corticolous.

Usnea exasperata (Müll.Arg.) Mot. - Piton de la Fournaise GF (B 60 0142010); St-Philippe GF (B 60 0135421). Corticolous.

Usnea gracilis Ach. - Forêt de Bébour PB40546, LG. Corticolous.

Usnea leprosa Motyka - Piton de la Fournaise GF (B 60 0142001). Corticolous.

Usnea rubicunda Stirt. - Forêt de Bébour PB40545; Plaine des Palmistes PB40896. Corticolous.

Usnea submollis J.Steiner - Piton de la Fournaise GF (B 60 0128915). Corticolous.

Xanthoparmelia phaeophana (Stirt.) Hale Cirque de Cilaos PB40202, US1498; Piton de la Fournaise GF (B 60 0135419). Saxicolous.

Xanthoparmelia salkiboensis Hale - Piton de la Fournaise GF (B 60 0135142). Saxicolous.

Xanthoparmelia subramigera (Gyeln.) Hale - Cirque de Salazie PB40956; Piton de la Fournaise GF (B 60 0135153). Saxicolous.

\section{Acknowledgements}

Field studies in Reunion were made possible with the help and advice from the Parc National de La Réunion, especially through the courtesy of Mr B. Lequette and Mr J. M. Pausé. Dr Cl. Ah-Peng and Prof. D. Strasberg of the University of La Réunion in Saint-Denis and Dr. J. Hivert of the Conservatoire Botanique National de Mascarin (St-Leu) were also very helpful. We thank them all most sincerely.

Our warm thanks are also due to Prof. Ted Ahti, Prof. Jack Elix, Prof. Per Magnus Jørgensen, Prof. Roland Moberg and Dr Mireia Giralt for their help with identification of several specimens.

Finally we thank Prof. Helmut Mayrhofer and both referees (Prof. Mark Seaward and anonymous) for their critical and helpful notes and suggestions.

\section{References}

Aнti, T. 2000. Cladoniaceae. - Flora Neotropica Monograph 78. Bronx, New York: Organization for Flora Neotropica and New York Botanical Garden Press.

Анті, T. \& Aртroot, A. 1992. Lichens of Madagascar: Cladoniaceae. - Cryptogamie, Bryologie, Lichénologie 13: 117-124.

Arup, U. \& van Den Boom, P. P. G. 2011. Three new dark-fruited Caloplaca species from Cape Verde. - Bibliotheca Lichenologica 106: 1-6.

Baloch, E., Lücking, R., Lumbsch, H. T. \& Wedin, M. 2010. Major clades and phylogenetic relationships between lichenized and non-lichenized lineages in Ostropales (Ascomycota: Lecanoromycetes). - Taxon 59: 1483-1494. 
BJerke, J. W. \& Sipman, H. J. M. 2007. New species and new records of Menegazzia (Parmeliaceae, lichenized ascomycetes) from Malaysia and Indonesia. - Botanical Journal of the Linnean Society 153: 489-499.

Bory de Saint-Vincent, J. B. G. M. 1804. Voyage dans les quatre principales îles des mers d’Afrique. - Paris: F. Buisson.

Bosser, J. 2007. Contribution to the study of the Orchidaceae from Madagascar, the Comoro islands and the Mascarenes. XXXVI. Descriptions of a new Cynorkis from Reunion and a new Angraecum from Madagascar. Adansonia 29: 13-17.

Burgess, N., D’Amico Hales, J., Underwood, E. \& Dinerstein, E. 2004. Terrestrial ecoregions of Africa and Madagascar. A conservation assessment. - Washington: Island Press.

CAdet, T. 1977. La végétation de l’île de la Réunion. Etude phytoécologique et phytosociologique. - PhD Thesis, Université d’Aix-Marseille. Cazal, St-Denis, La Réunion.

Calvelo, S. 1996. Noteworthy reports on Anzia (lichenized Ascomycotina) from southern South-America. Mycotaxon 58: 147-156.

Caujapé-Castells, J., Tye, A., Crawford, D. J., Santos-Guerra, A., Sakai, A., Beaver, K., Lobin, W., Florens, F. B. V., Moura, M., Jardim, R., Gómes, I. \& Kueffer, C. 2010. Conservation of oceanic island floras: Present and future global challenges. - Perspectives in Plant Ecology, Evolution and Systematics 12: 107-129.

Chene, A. \& Hume, J. 2008. Lost land of the dodo. An ecological history of Mauritius, Réunion \& Rodrigues. London: T \& AD Poyser.

Delise, D. 1825. Histoire de lichens. Genre Sticta. - Caen.

DelmaIl, D. 2009. Selaginella bifida sp. nov. (Selaginellaceae: Pteridophyta) from Rodrigues Island, Mauritius. Nordic Journal of Botany 27: 178-181.

DEs AbBAyes, H. 1961. Lichens récoltés à Madagascar et à La Réunion (Mission H. des Abbayes, 1956). I. Introduction. II. Parméliacées. - Mémoires de l'Institut Scientifique de Madagascar, Sér. B, 10: 81-121.

Doumenge, C. \& RenARD, Y. 1989. La conservation des écosystèmes forestiers de l'île de La Réunion. - Gland: IUCN. ELIX, J. A. 2010. Additional lichen records from Australia 72. - Australasian Lichenology 66: 60-69.

ERTz, D. 2009. Revision of the corticolous Opegrapha species from the Paleotropics. - Bibliotheca Lichenologica 102: $1-176$.

Ertz, D. \& Tehler, A. 2011. The phylogeny of Arthoniales (Pezizomycotina) inferred from nucLSU and RPB2 sequences. - Fungal Diversity 46 (doi:10.1007/s13225-010-0080-y; erratum at http://dx.doi.org/10.1007/s13225011-0100-6).

Ertz, D., Elix, J. \& Grube, M. 2010. Arthonia borbonica (Ascomycota, Arthoniales), a new species from La Réunion. - Plant Ecology and Evolution 143: 222-224.

Ferraro, L. I. \& Lücking, R. 2005. The genus Gomphillus (Ostropales: Gomphillaceae) in the Americas, with the new species Gomphillus pedersenii from Argentina. - The Bryologist 108: 491-496.

Frisch, A. \& Thor, G. 2009. Crypthonia, a new genus of byssoid Arthoniaceae (lichenised Ascomycota). Mycological Progress 9: 281-303.

Galloway, D. J. 2007. Flora of New Zealand: Lichens, including lichen-forming and lichenicolous fungi. - Lincoln, New Zealand: Manaaki Whenua Press, Landcare Research.

Galloway, D. J. 2008. Lichen biogeography. - In: NASH, T. H. III (ed.): Lichen biology. Second edition. Pp. 315 -335. - Cambridge: Cambridge University Press.

Gerlach, J. 2004. Giant tortoises of the Indian Ocean. The genus Dipsochelys inhabiting the Seychelles Islands and the extinct giants of Madagascar and the Mascarenes. - Frankfurt am Main: Edition Chimaira.

Giralt, M., Paz-Bermúdez, G. \& Elix, J. A. 2009. New data on Sculptolumina japonica (Physciaceae). - The Bryologist 112: 397-403.

Gueidan, C., Savić, S., Thüs, H., Roux, C., Keller, C., Tibell, L., Prieto, M., HeiĐmarsson, S., Breuss, O., Orange, A., Fröberg, L., Amtoft Wynns, A., Navarro-Rosinés, P., Krzewicka, B., Pykälä, J., Grube, M. \& Lutzoni, F. 2009. Generic classification of the Verrucariaceae (Ascomycota) based on molecular and morphological evidence: recent progress and remaining challenges. - Taxon 58: 184-208.

Hafellner, J. 1997. A world monograph of Brigantiaea (lichenized Ascomycotina, Lecanorales). - Symbolae Botanicae Upsalienses. Acta Universitatis Upsaliensis 32: 35-74.

Hestmark, G., Miądlikowska, J., Kauff, F., Raker, E., Molnar, K. \& Lutzoni, F. 2011. Single origin and subsequent diversification of central Andean endemic Umbilicaria. - Mycologia 103: 45-56.

JørGensen, P. M. 2001. New species and records of the lichen family Pannariaceae from Australia. - Bibliotheca Lichenologica 78: 109-139.

Jørgensen, P. M. 2003. Notes on African Pannariaceae (lichenized Ascomycetes). - The Lichenologist 35: 11-20.

Jørgensen, P. M. 2004. Further contributions to the Pannariaceae (lichenized Ascomycetes) of the Southern Hemisphere. - Bibliotheca Lichenologica 88: 229-253.

Jørgensen, P. M. \& Palice, Z. 2010. Additions to the lichen family Pannariaceae in Ecuador. - Nordic Journal of Botany 28: 623-628. 
Jørgensen, P. M. \& Schumm, F. 2010. Parmeliella borbonica, a new lichen species from Réunion. - The Lichenologist 42: 697-700.

Jørgensen, P. M., van den Boom, P. P. G. \& Sérusiaux, E. 2009. Notes on the lichen genus Erioderma in La Réunion. - Cryptogamie, Mycologie 30: 263-268.

KALB, K. 2004. New or otherwise interesting lichens II. - Bibliotheca Lichenologica 88: 301-329.

KALB, K. 2009. New taxa and new records of thelotremoid Graphidaceae. - Herzogia 22: 17-42.

Kalb, K., Archer, A. W., Sutjaritturakan, J. \& Boonpragob, K. 2009a. New or otherwise interesting lichens V. - Bibliotheca Lichenologica 99: 225-246.

Kalb, K., Buaruang, K., Papong, K. \& Boonprabog, K. 2009b. New or otherwise interesting lichens from the tropics, including the lichen genus Ramboldia in Thailand. - Mycotaxon 110: 109-123.

Knox, M. D. E. 1982. Three new species of Hypotrachyna from South Africa. - The Bryologist 85: 118-121.

Komposch, H. \& Hafellner, J. 1999. List of lichenized fungi so far observed in the tropical lowland rain forest plot Surumoni (Venezuela, Estado Amazonas). - Fritschiana (Graz) 19: 1-10.

KroG, H. 2000. Corticolous macrolichens of low montane rainforests and moist woodlands of eastern Tanzania. Sommerfeltia 28: 1-75.

Lacroix, A. 1917. Le Naturaliste Bory de Saint-Vincent. - Revue scientifique [55e année] 8 [Éloge du savant prononcé en octobre 1916 à l’Académie des Sciences]

LAmb, I. M. 1977. A conspectus of the lichen genus Stereocaulon (Schreb.) Hoffm. - Journal of the Hattori Botanical Laboratory 43: 191-355.

LEROND M. 1998. Qualité de l'air de l’Ile de La Réunion. Première approche. - Rapport au Ministère des Affaires sociales, de la santé et de la ville, et à l'Observtoire régional de la santé de La Réunion.

Losos, J. B. \& Ricklefs, R. E. 2009. Adaptation and diversification on islands. - Nature 457: 830-836.

LÜCKING, R. \& SÉRusiauX, E. 2005. Gomphillus morchelloides (Ostropales: Gomphillaceae), a new lichen species from Chile and Papua New Guinea. - The Bryologist 108: 487-490.

Lücking, R., Rivas Plata, E., Chaves, J. L., Umaña, L. \& Sipman, H. J. M. 2009. How many tropical lichens are there ... really ? - Bibliotheca Lichenologica 100: 399-418.

Lumbsch, T. H. \& LeavitT, S. D. 2011. Goodbye morphology? A paradigm shift in the delimitation of species in lichenized fungi. - Fungal Diversity 50: 59-72.

LumbSCH, T. H. et al. 2011. One hundred new species of lichenized fungi: a signature of undiscovered global diversity. - Phytotaxa 18: 1-127.

McCarthy, P. M. \& Elix, J. A. 1996. Myeloconis, a new genus of pyrenocarpous lichens from the tropics. - The Lichenologist 28: 401-414.

Mangold, A., Martin, M. P., Lücking, R. \& Lumbsch, T. 2008. Molecular phylogeny suggests synonymy of Thelotremataceae with Graphidaceae (Ascomycota: Ostropales). - Taxon 57: 476-486.

Mathey, A. 1971. Contribution à l'étude du genre Siphula (lichens) en Afrique. - Nova Hedwigia 22: 795 -878.

Nelsen, M. P., Lücking, R., Grube, M., Mbatchou, J. S., Muggia, L., Rivas Plata, E. \& Lumbsch, H. T. 2009. Unravelling the phylogenetic relationships of lichenised fungi in Dothideomyceta. - Studies in Mycology 64: $135-144$.

North, S. G., Bullock, D. J. \& Dulloo, M. E. 1994. Changes in the vegetation and reptile populations of Round Island, Mauritius, following eradication of rabbits. - Biological Conservation 67: 21-28.

NyLANDER, W. 1859. Lichenes exotici, lichenes in regionibus exoticis quibusdam vigentes exponit synopticis enumerationibus. - Annales des Sciences Naturelles 11: 205-264.

Orange, A., James, P. W. \& White, F. J. 2001. Microchemical methods for the identification of lichens. - London: British Lichen Society.

Printzen, C., Ekman, S. \& Tønsberg, T. 2003. Phylogeography of Cavernularia hultenii: evidence of slow genetic drift in a widely disjunct lichen. - Molecular Ecology 12: 1473-1486.

Rivas Plata, E. \& Lumbsch, H. T. 2011. Parallel evolution and phenotypic divergence in lichenized fungi: A case study in the lichen-forming fungal family Graphidaceae (Ascomycota: Lecanoromyctes: Ostropales). - Molecular Phylogenetics and Evolution 61: 45-63.

Ronhede, S., Johansson, S. \& Søchting, U. 2003. A contribution to the foliicolous lichen flora of La Réunion. Cryptogamie, Mycologie 24: 67-71.

Sauvageau, C. 1908. Bory de Saint-Vincent, d'après sa correspondance publiée par M. Lauzun. - Journal de Botanique, 2e Série, 1: 198-222.

Schmull, M., Miądlikowska, J., Pelzer, M., Stocker-Wörgötter, E., Hofstetter, V., Fraker, E., Hodkinson, B. P., Reeb, V., Kukwa, M., Lumbsch, H. T., Kauff, F. \& Lutzoni, F. 2011. Phylogenetic affiliations of members of the heterogeneous lichen-forming fungi of the genus Lecidea sensu Zahlbruckner (Lecanoromycetes, Ascomycota). - Mycologia 103: 983-1003.

Seaward, M. R. D. \& Aptroot, A. 2009. Checklist of lichens for the Seychelles group. - Bibliotheca Lichenologica 99: 335-366. 
SÉrusiaux, E. 1977. Quelques lichens foliicoles récoltés à La Réunion (Afrique, Océan Indien). - Bulletin de la Société Royale de Botanique de Belgique 110: 39-41.

SÉrusiaux, E. 1997: Sporopodiopsis, a new genus of lichens (Ectolechiaceae) from S-E Asia. - Abstracta Botanica 21: $145-152$.

Sérusiaux, E., Brand, A. M., Fischer, E., Killmann, D., van den Boom, P. P. G. \& Ertz, D. 2009. A new species of Melanotopelia (Graphidaceae) from Africa. - The Lichenologist 41: 243-247.

SÉrusiaux, E., van den Boom, P. P. G. \& Ertz, D. 2010a. A two-gene phylogeny shows the lichen genus Niebla (Lecanorales) is endemic to the New World and does not occur in Macaronesia nor in the Mediterranean basin. Fungal Biology 114: 528-537.

Sérusiaux, E., Brand, A. M., Motiejūnaité, J., Orange, A. \& Coppins, B. J. 2010b. Lecidea doliiformis belongs to Micarea, Catillaria alba to Biatora, and Biatora ligni-mollis occurs in western Europe. - The Bryologist 113 : 333-344.

Sérusiaux, E., Villareal, A. J. C., Wheeler, T. \& Goffinet, B. 2011. Recent origin, active speciation and dispersal for the lichen genus Nephroma in Macaronesia. - Journal of Biogeography 38: 1138-1151.

Shapiro, B., Sibthorpe, D., Rambaut, A., Austin, J., Wragg, G. M., Bininda-Emonds, O. R. P., Lee, P. L. M. \& Cooper, A. 2002. Flight of the dodo. - Science 295: 1683.

Sipman, H. J. M. 1983. A monograph of the lichen family Megalosporaceae. - Bibliotheca Lichenologica 18: 1-241 + plates.

Stenroos, S. 1991. Status of four species of Cladonia endemic to the Madagascan Region. - Annales Botanici Fennici 28: 107-110.

Tehler, A., Irestedt, M., Wedin, M. \& Ertz, D. 2009. Origin, evolution and taxonomy of American Roccella (Roccellaceae, Ascomycetes). - Systematics and Biodiversity 7: 307-317

Tehler, A., Irestedt, M., Wedin, M. \& Ertz, D. 2010. The Old World Roccella species outside Europe and Macaronesia: taxonomy, evolution and phylogeny. - Systematics and Biodiversity 8: 223-246

Timdal, E. \& Krog, H. 2001. Further studies on African species of the lichen genus Phyllopsora (Lecanorales). Mycotaxon 77: 57-89.

van DEN Boom, P. P. G., Ertz, D., Brand, M. \& SÉrusiaux, E. 2011. Syncesia mascarena (Roccellaceae), a new species from La Réunion (Indian Ocean). - Opuscula Philolichenum 9: 5-10.

Verdon, D. \& Rambold, G. 1998. A new species in the genus Solenopsora (Catillariaceae, Lecanorales). - Mycotaxon 69: $399-408$.

VĚZDA, A. 1979: Flechtensystematische Studien XI. Beiträge zur Kenntnis der Familie Asterothyriaceae (Discolichenes). - Folia Geobotanica et Phytotaxonomica 14: 43-94.

VĚzDA, A. \& Malcolm, W. M. 1997. Gyalideopsis zeylandica (Gomphillaceae), a new saxicolous species from New Zealand. - Australasian Lichenological Newsletter 40: 20-21.

Wirtz, N., Printzen, C. \& Lumbsch, H. T. 2008. The delimitation of Antarctic and bipolar species of neuropogonoid Usnea (Ascomycota, Lecanorales): a cohesion approach of species recognition for the Usnea perpusilla complex. - Mycological Research 112: 472-484.

Yoshimura, I., Sipman, H. J. M. \& Aptroot, A. 1995: The lichen genus Anzia in New Guinea. - Bibliotheca Lichenologica 58: 439-469.

Manuscript accepted: 10 October 2011.

\section{Addresses of the authors}

Pieter P. G. van den Boom, Arafura 16, 5691 JA, Son, The Netherlands.

E-mail: pvdboom@kpnmail.nl

Maarten Brand, Klipperwerf 5, 2317 DX, Leiden, The Netherlands.

E-mail: a.maartenbrand@gmail.com

Damien Ertz, National Botanic Garden of Belgium, Domaine de Bouchout, 1860 Meise, Belgium. E-mail: damien.ertz@br.fgov.be

Klaus Kalb, Lichenologisches Institut Neumarkt, Im Tal 12, 92318 Neumarkt, Germany \& University of Regensburg, Institute for Botany, Universitätsstraße 31, 93040 Regensburg, Germany. E-mail: klaus.kalb@arcor.de 
Nicolas Magain, Evolution and Conservation Biology Unit, University of Liège, Sart Tilman B22, 4000 Liège, Belgium. E-mail: Nicolas.Magain@doct.ulg.ac.be

Didier Masson, rue des Flamboyants 386, 40600 Biscarrosse, France.

E-mail: di.masson@wanadoo.fr

Ulf Schiefelbein, Blücherstraße 71, 18055 Rostock, Germany.

E-mail: ulf.schiefelbein@gmx.de

Harrie J. M. Sipman, Freie Universität Berlin, Botanischer Garten und Botanisches Museum, Königin-Luise-Straße 6-8, 14195 Berlin, Germany. E-mail: h.sipman@bgbm.org

Emmanuël Sérusiaux, Evolution and Conservation Biology Unit, University of Liège, Sart Tilman B22, 4000 Liège, Belgium. E-mail: E.Serusiaux@ulg.ac.be 
